\title{
Some Interval 2-Tuple Linguistic Harmonic Mean Operators and Their Application in Material Selection
}

\author{
Meng-Meng Shan, ${ }^{1}$ Jian-Xin You, ${ }^{2}$ and Hu-Chen Liu ${ }^{1,2}$ \\ ${ }^{1}$ School of Management, Shanghai University, Shanghai 200444, China \\ ${ }^{2}$ School of Economics and Management, Tongji University, Shanghai 200092, China \\ Correspondence should be addressed to Hu-Chen Liu; huchenliu@foxmail.com
}

Received 13 April 2016; Accepted 30 June 2016

Academic Editor: Charles C. Sorrell

Copyright (c) 2016 Meng-Meng Shan et al. This is an open access article distributed under the Creative Commons Attribution License, which permits unrestricted use, distribution, and reproduction in any medium, provided the original work is properly cited.

\begin{abstract}
We investigate the multiple attribute group material selection problems in which the attribute values take the form of interval 2-tuple linguistic information. Firstly, some operational laws and possibility degree of interval 2-tuple linguistic variables are introduced. Then, we develop some interval 2-tuple linguistic aggregation operators called interval 2-tuple hybrid harmonic mean (ITHHM) operator, induced interval 2-tuple ordered weighted harmonic mean (I-ITOWHM) operator, and induced interval 2-tuple hybrid harmonic mean (I-ITHHM) operator and study some desirable properties of the I-ITOWHM operator. In particular, all these operators can be reduced to aggregate 2-tuple linguistic variables. Based on the I-ITHHM and the ITWHM (interval 2-tuple weighted harmonic mean) operators, an approach to multiple attribute group decision-making with interval 2-tuple linguistic information is proposed. Finally, a practical application to material selection problem is given to verify the developed approach and to demonstrate its practicality and effectiveness.
\end{abstract}

\section{Introduction}

The 2-tuple linguistic representation model, characterized by a linguistic term and a numeric value, was developed by Herrera and Martínez [1] based on the concept of symbolic translation. It has exact characteristic in linguistic information processing and can effectively avoid information distortion and loss which occur formerly in the linguistic computing process. In many real decision-making cases, experts tend to give opinions according to their experience and knowledge, and it is more suitable to provide assessments by means of linguistic terms rather than numerical ones $[2,3]$, due to the complexity of the objects and the vagueness of human thinking. Therefore, many researchers have investigated linguistic multiple attribute group decision-making (MAGDM) problems and proposed lots of methods to deal with linguistic evaluation information. These linguistic computational models can be mainly classified into three types $[3,4]$ : the method based on membership functions, the method based on linguistic symbols, and the method based on linguistic 2-tuples. However, one important weakness of the former two linguistic computational models is that they performed the retranslation step as an approximation process to express the results in the original expression domain (initial term set) thus provoking a lack of accuracy [5]. In contrast, the 2-tuple linguistic method is a symbolic model that extends the use of indexes modifying the fuzzy linguistic approach representation by adding a parameter to the basic linguistic representation in order to improve the accuracy of the linguistic computations and the interpretability of the results. Due to its distinguished power and efficiency in dealing with linguistic assessments of decision-makers, the 2-tuple linguistic model has been extensively applied to decision-making processes since its appearance [6-10].

In recent years, much progress has been made in research relating to 2-tuple aggregation operators since information aggregation plays a significant part in the MAGDM process. For example, Herrera and Martínez [1] defined the 2-tuple arithmetic mean operator, the 2-tuple weighted averaging operator, and the 2-tuple ordered weighted averaging operator. Wei [11] proposed the extended 2-tuple weighted geometric (ET-WG) and the extended 2-tuple ordered weighted 
geometric (ET-OWG) operators and Wei [12] developed some generalized aggregating operators with 2-tuple linguistic information, such as the generalized 2-tuple weighted average (G-2TWA), the generalized 2-tuple ordered weighted average (G-2TOWA), and the induced generalized 2-tuple ordered weighted average (IG-2TOWA) operators. Yang and Chen [13] considered the linguistic group decision-making problem with interdependent attributes and introduced some 2 -tuple linguistic aggregation operators by using the Choquet integral: the 2-tuple correlated averaging (TCA) operator, the 2-tuple correlated geometric (TCG) operator, and the generalized 2-tuple correlated averaging (GTCA) operator. $\mathrm{Xu}$ and Wang [14] developed the 2-tuple linguistic power average (2TLPA), the 2-tuple linguistic weighted power average (2TLWPA), and the 2-tuple linguistic power ordered weighted average (2TLPOWA) operators and studied their desired properties, such as commutativity, idempotency, and boundary. Wan [15] developed some hybrid arithmetic aggregation operators with 2-tuple linguistic information, involving the 2-tuple hybrid weighted arithmetic average (THWA) operator, the 2-tuple hybrid linguistic weighted arithmetic average (T-HLWA) operator, and the extended 2-tuple hybrid linguistic weighted arithmetic average (ETHLWA) operator. Recently, Sonia et al. [16] introduced some linguistic aggregation operators with conservation of interaction between criteria, which include the 2-tuple Choquet integral averaging (TCIA) operator, the 2-tuple ordered Choquet integral averaging (TOCIA) operator, and the combined 2-tuple Choquet integral averaging operator. Considering the interactive phenomenon among experts (or attributes) in MAGDM, Lin et al. [17] proposed a generalized interval 2-tuple linguistic Shapley chi-square averaging operator for facility location selection. Li and Liu [18] proposed some new aggregation operators of 2-tuple linguistic information based on Heronian mean, and Ju et al. [19] gave some new Shapley 2tuple linguistic Choquet aggregation operators for MAGDM. The linguistic proportional 2-tuple power average operator was introduced by Jiang et al. [20] to aggregate linguistic values of unbalanced linguistic term sets considering the relationship among the aggregated values. Besides, the 2tuple linguistic extended Bonferroni mean (EBM) aggregation operators and the 2-tuple linguistic partition Bonferroni mean (PBM) aggregation operators were developed in [21] and [22], respectively, for dealing with MAGDM problems.

In some situations, however, the input arguments take the form of interval 2-tuple linguistic values because of time pressure, lack of knowledge or data, and decisionmakers' limited attention and information processing capabilities [23-25]. Furthermore, all decision-makers cannot easily express their evaluations on the established criteria with one given linguistic term set. That is, some decisionmakers feel that the cardinality of the linguistic term set is too small to fully express their judgments on the alternatives, while others think it is so big that the evaluations on the alternatives are out of their ability. Therefore, Zhang [26] introduced the interval 2-tuple linguistic representation model and developed some interval 2-tuple linguistic aggregation operators, such as the interval 2-tuple weighted average (ITWA) and the interval 2-tuple ordered weighted average (ITOWA) operators. Zhang [27] further developed some new interval 2-tuple linguistic aggregation operators, including the interval 2-tuple weighted geometric (ITWG), the interval 2-tuple ordered weighted geometric (ITOWG), the generalized interval 2-tuple weighted average (GITWA), and the generalized interval 2-tuple ordered weighted average (GITOWA) operators. Liu et al. [28] put forward some Bonferroni mean operators under the interval-valued 2tuple linguistic context and investigated their properties and special cases. Wu et al. [29] presented the 2-tuple linguistic generalized power average (2TLGPA), the 2-tuple linguistic generalized power ordered weighted average (2TLGPOWA), and the interval 2-tuple linguistic generalized power average (I2TLGPA) operators for MAGDM. Motivated by the idea of harmonic mean operators [30,31], in this paper, we develop some interval 2-tuple linguistic harmonic mean operators, such as the interval 2-tuple hybrid harmonic mean (ITHHM) operator, the induced interval 2-tuple ordered weighted harmonic mean (I-ITOWHM) operator, and the induced interval 2-tuple hybrid harmonic mean (I-ITHHM) operator. And a new approach based on the I-ITHHM and the interval 2-tuple weighted harmonic mean (ITWHM) operators for MAGDM with interval 2-tuple linguistic information is presented.

The remainder of this paper is set out as follows. In Section 2, we introduce some basic concepts and operation laws of interval 2-tuple linguistic variables. In Section 3, we develop some new aggregation operators called the ITHHM operator, the I-ITOWHM operator, and the IITHHM operator and investigate some desirable properties of the I-ITOWHM operator. In Section 4, we develop an approach based on the I-ITHHM and the ITWHM operators to interval 2-tuple linguistic MAGDM. A material selection example is given in Section 5 to verify the developed approach and to demonstrate its feasibility and practicality. Finally, conclusions and future directions are provided in Section 6.

\section{Preliminaries}

2.1. 2-Tuple Linguistic Variables. A linguistic variable is a variable whose values are expressed in linguistic terms. In other words, it is a variable whose values are not numbers but words or sentences in a natural or artificial language. The concept of linguistic variables is very useful in dealing with situations which are too complex or too ill defined to be reasonably described by traditional quantitative expressions [32]. For identifying the diversity of each evaluation item and facilitating the computation, linguistic terms often possess some characteristics such as finite set, odd cardinality, semantic symmetric, ordinal level, and compensative operation [33]. Let $S=\left\{s_{i} \mid i=0,1, \ldots, g\right\}$ be a linguistic term set with odd cardinality, where $s_{i}$ represents a possible value for a linguistic variable. It is required that the linguistic term set should satisfy the following characteristics [1]:

(1) Negation operator: $\operatorname{Neg}\left(s_{i}\right)=s_{j}$ such that $j=g-i$.

(2) The set which is ordered: $s_{i}>s_{j}$, if $i>j$.

(3) Max operator: $\max \left(s_{i}, s_{j}\right)=s_{i}$, if $s_{i} \geq s_{j}$.

(4) Min operator: $\min \left(s_{i}, s_{j}\right)=s_{i}$, if $s_{i} \leq s_{j}$. 
The 2-tuple linguistic representation model was firstly presented by Herrera and Martínez [1] based on the concept of symbolic translation. It is used to represent the linguistic information by means of a linguistic 2-tuple, $(s, \alpha)$, where $s$ is a linguistic term from the predefined linguistic term set $S$ and $\alpha$ is a numerical value representing the symbolic translation. In the classical 2-tuple linguistic approach, the range of $\beta$ value is relevant to the granularity of the linguistic term sets. Here, $\beta$ is the result of an aggregation of the indices of a set of labels assessed in the linguistic term set $S$. To overcome this restriction, Tai and Chen [33] further proposed a generalized 2-tuple linguistic model and translation functions.

Definition 1. Let $S=\left\{s_{0}, s_{1}, \ldots, s_{g}\right\}$ be a linguistic term set and let $\beta \in[0,1]$ be a value representing the result of a symbolic aggregation operation. Then, the generalized translation function $\Delta$ used to obtain the 2-tuple equivalent to $\beta$ can be defined as follows [33]:

$$
\begin{aligned}
& \Delta:[0,1] \longrightarrow S \times\left[-\frac{1}{2 g}, \frac{1}{2 g}\right) \\
& \Delta(\beta)=\left(s_{i}, \alpha\right), \\
& \text { with } \begin{cases}s_{i}, & i=\operatorname{round}(\beta \cdot g) \\
\alpha=\beta-\frac{i}{g}, & \alpha \in\left[-\frac{1}{2 g}, \frac{1}{2 g}\right),\end{cases}
\end{aligned}
$$

where round $(\cdot)$ is the usual rounding operation. The interval of $\alpha$ is determined by the number of linguistic terms in $S$. For example, if $S$ contains seven linguistic terms, then $g=6$ and $\alpha \in[-0.083,0.083)$.

Definition 2. Let $S=\left\{s_{0}, s_{1}, \ldots, s_{g}\right\}$ be a linguistic term set and let $\left(s_{i}, \alpha\right)$ be a 2 -tuple. There exists a function $\Delta^{-1}$, which is able to convert the 2-tuple into its equivalent numerical value $\beta \in[0,1]$. The reverse function $\Delta^{-1}$ is defined as follows [33]:

$$
\begin{aligned}
\Delta^{-1}: S \times\left[-\frac{1}{2 g}, \frac{1}{2 g}\right) & \longrightarrow[0,1], \\
\Delta^{-1}\left(s_{i}, \alpha\right) & =\frac{i}{g}+\alpha=\beta .
\end{aligned}
$$

It is obvious that the conversion of a linguistic term into a linguistic 2 -tuple consists of adding a value 0 as symbolic translation [1]:

$$
s_{i} \in S \Longrightarrow\left(s_{i}, 0\right) \text {. }
$$

2.2. Interval 2-Tuple Linguistic Variables. The interval 2-tuple linguistic representation model was put forward by Zhang [26] as a generalization of the 2-tuple linguistic variable.

Definition 3. Let $S=\left\{s_{0}, s_{1}, \ldots, s_{g}\right\}$ be a linguistic term set. An interval 2-tuple linguistic variable is composed of two 2tuples, denoted by $\left[\left(s_{i}, \alpha_{i}\right),\left(s_{j}, \alpha_{j}\right)\right]$, where $\left(s_{i}, \alpha_{i}\right) \leq\left(s_{j}, \alpha_{j}\right)$, and $s_{i}\left(s_{j}\right)$ and $\alpha_{i}\left(\alpha_{j}\right)$ represent the linguistic label of the linguistic term set $S$ and symbolic translation, respectively.
The interval 2-tuple that expresses the equivalent information to an interval value $\left[\beta_{1}, \beta_{2}\right]\left(\beta_{1}, \beta_{2} \in[0,1], \beta_{1} \leq \beta_{2}\right)$ is derived by the following function $[26,27]$ :

$$
\begin{array}{r}
\Delta\left[\beta_{1}, \beta_{2}\right]=\left[\left(s_{i}, \alpha_{i}\right),\left(s_{j}, \alpha_{j}\right)\right] \\
\text { with } \begin{cases}s_{i}, & i=\operatorname{round}\left(\beta_{1} \cdot g\right) \\
s_{j}, & j=\operatorname{round}\left(\beta_{2} \cdot g\right) \\
\alpha_{i}=\beta_{1}-\frac{i}{g}, & \alpha_{i} \in\left[-\frac{1}{2 g}, \frac{1}{2 g}\right) \\
\alpha_{j}=\beta_{2}-\frac{j}{g}, & \alpha_{j} \in\left[-\frac{1}{2 g}, \frac{1}{2 g}\right) .\end{cases}
\end{array}
$$

On the contrary, there is always a function $\Delta^{-1}$ such that an interval 2-tuple can be converted into an interval value $\left[\beta_{1}, \beta_{2}\right]\left(\beta_{1}, \beta_{2} \in[0,1], \beta_{1} \leq \beta_{2}\right)$ as follows:

$$
\Delta^{-1}\left[\left(s_{i}, \alpha_{i}\right),\left(s_{j}, \alpha_{j}\right)\right]=\left[\frac{i}{g}+\alpha_{i}, \frac{j}{g}+\alpha_{j}\right]=\left[\beta_{1}, \beta_{2}\right] .
$$

In particular, if $s_{i}=s_{j}$ and $\alpha_{i}=\alpha_{j}$, then the interval 2-tuple linguistic variable reduces to a 2 -tuple linguistic variable.

Definition 4. Consider any three interval 2-tuples $\widetilde{a}=[(s, \alpha)$, $(t, \varepsilon)], \widetilde{a}_{1}=\left[\left(s_{1}, \alpha_{1}\right),\left(t_{1}, \varepsilon_{1}\right)\right]$, and $\widetilde{a}_{2}=\left[\left(s_{2}, \alpha_{2}\right),\left(t_{2}, \varepsilon_{2}\right)\right]$ and let $\lambda \in[0,1]$; then, their operations can be defined as follows [34]:

$$
\begin{aligned}
\tilde{a}_{1} & \otimes \widetilde{a}_{2}=\left[\left(s_{1}, \alpha_{1}\right),\left(t_{1}, \varepsilon_{1}\right)\right] \otimes\left[\left(s_{2}, \alpha_{2}\right),\left(t_{2}, \varepsilon_{2}\right)\right] \\
& =\Delta\left[\Delta^{-1}\left(s_{1}, \alpha_{1}\right) \cdot \Delta^{-1}\left(s_{2}, \alpha_{2}\right), \Delta^{-1}\left(t_{1}, \varepsilon_{1}\right)\right. \\
& \left.\cdot \Delta^{-1}\left(t_{2}, \varepsilon_{2}\right)\right] ; \\
\widetilde{a}_{1} & \oplus \widetilde{a}_{2}=\left[\left(s_{1}, \alpha_{1}\right),\left(t_{1}, \varepsilon_{1}\right)\right] \oplus\left[\left(s_{2}, \alpha_{2}\right),\left(t_{2}, \varepsilon_{2}\right)\right] \\
& =\Delta\left[\Delta^{-1}\left(s_{1}, \alpha_{1}\right)+\Delta^{-1}\left(s_{2}, \alpha_{2}\right), \Delta^{-1}\left(t_{1}, \varepsilon_{1}\right)\right. \\
& \left.+\Delta^{-1}\left(t_{2}, \varepsilon_{2}\right)\right] ; \\
\tilde{a}^{\lambda} & =([(s, \alpha),(t, \varepsilon)])^{\lambda} \\
& =\Delta\left[\left(\Delta^{-1}(s, \alpha)\right)^{\lambda},\left(\Delta^{-1}(t, \varepsilon)\right)^{\lambda}\right] ; \\
\lambda \widetilde{a} & =\lambda[(s, \alpha),(t, \varepsilon)]=\Delta\left[\lambda \Delta^{-1}(s, \alpha), \lambda \Delta^{-1}(t, \varepsilon)\right] .
\end{aligned}
$$

Motivated by the formulas proposed by Xu $[35,36]$, the comparison of linguistic information represented by interval 2-tuples is implemented on the basis of the possibility degree of the interval 2-tuple linguistic variables. It can be defined as follows.

Definition 5. Let $\widetilde{a}_{1}=\left[\left(s_{1}, \alpha_{1}\right),\left(t_{1}, \varepsilon_{1}\right)\right]$ and $\tilde{a}_{2}=\left[\left(s_{2}, \alpha_{2}\right)\right.$, $\left.\left(t_{2}, \varepsilon_{2}\right)\right]$ be two interval 2-tuples, and let $h\left(\widetilde{a}_{1}\right)=\Delta^{-1}\left(t_{1}, \varepsilon_{1}\right)$ - 
$\Delta^{-1}\left(s_{1}, \alpha_{1}\right)=\delta_{1}-\beta_{1}$ and let $h\left(\widetilde{a}_{2}\right)=\Delta^{-1}\left(t_{2}, \varepsilon_{2}\right)-\Delta^{-1}\left(s_{2}, \alpha_{2}\right)=$ $\delta_{2}-\beta_{2}$; then, the possibility degree of $\widetilde{a}_{1} \geq \widetilde{a}_{2}$ is defined as

$$
\begin{aligned}
p & \left(\tilde{a}_{1} \geq \tilde{a}_{2}\right) \\
& =\max \left\{1-\max \left(\frac{\delta_{2}-\beta_{1}}{h\left(\tilde{a}_{1}\right)+h\left(\tilde{a}_{2}\right)}, 0\right), 0\right\} .
\end{aligned}
$$

Similarly, the possibility degree of $\tilde{a}_{2} \geq \tilde{a}_{1}$ is defined as

$$
\begin{aligned}
p & \left(\widetilde{a}_{2} \geq \tilde{a}_{1}\right) \\
& =\max \left\{1-\max \left(\frac{\delta_{1}-\beta_{2}}{h\left(\tilde{a}_{1}\right)+h\left(\tilde{a}_{2}\right)}, 0\right), 0\right\} .
\end{aligned}
$$

From Definition 5, we can easily get the following results:

(1) $0 \leq p\left(\widetilde{a}_{1} \geq \widetilde{a}_{2}\right) \leq 1,0 \leq p\left(\widetilde{a}_{2} \geq \widetilde{a}_{1}\right) \leq 1$;

(2) $p\left(\widetilde{a}_{1} \geq \widetilde{a}_{2}\right)+p\left(\widetilde{a}_{2} \geq \widetilde{a}_{1}\right)=1$. In particular, $p\left(\widetilde{a}_{1} \geq\right.$ $\left.\tilde{a}_{1}\right)=p\left(\tilde{a}_{2} \geq \tilde{a}_{2}\right)=0.5$.

\section{Interval 2-Tuple Linguistic Harmonic Mean Operators}

\subsection{Interval 2-Tuple Hybrid Harmonic Mean Operator}

Definition 6. Let WHM: $\left(R^{+}\right)^{n} \rightarrow R^{+}$, if

$$
\operatorname{WHM}\left(a_{1}, a_{2}, \ldots, a_{n}\right)=\frac{1}{\sum_{i=1}^{n} w_{i} / a_{i}},
$$

where $a_{i}(i=1,2, \ldots, n)$ is a collection of positive real numbers, $w=\left(w_{1}, w_{2}, \ldots, w_{n}\right)^{T}$ is the weight vector of $a_{i}(i=1,2$, $\ldots, n)$, with $w_{i} \in[0,1]$ and $\sum_{i=1}^{n} w_{i}=1$, and $R^{+}$is the set of all positive real numbers; then, WHM is called the weighted harmonic mean operator [37].

Zhang [27] extended the WHM operator to accommodate the situations where the input arguments are interval 2tuple linguistic variables. For convenience, we let $\widetilde{S}$ be the set of all interval 2-tuple linguistic variables.

Definition 7. Let $\tilde{a}_{i}=\left[\left(s_{i}, \alpha_{i}\right),\left(t_{i}, \varepsilon_{i}\right)\right](i=1,2, \ldots, n)$ be a set of interval 2-tuples, and let ITWHM: $\widetilde{S}^{n} \rightarrow \widetilde{S}$, if

$$
\begin{aligned}
& \operatorname{ITwhM}\left(\tilde{a}_{1}, \tilde{a}_{2}, \ldots, \tilde{a}_{n}\right)=\frac{1}{\bigoplus_{i=1}^{n}\left(w_{i} / \tilde{a}_{i}\right)} \\
& =\Delta\left[\frac{1}{\sum_{i=1}^{n} w_{i} /\left(\Delta^{-1}\left(s_{i}, \alpha_{i}\right)\right)}, \frac{1}{\sum_{i=1}^{n} w_{i} /\left(\Delta^{-1}\left(t_{i}, \varepsilon_{i}\right)\right)}\right],
\end{aligned}
$$

where $w=\left(w_{1}, w_{2}, \ldots, w_{n}\right)^{T}$ is the weight vector of $\widetilde{a}_{i}(i=$ $1,2, \ldots, n)$, with $w_{i} \in[0,1]$ and $\sum_{i=1}^{n} w_{i}=1$; then, ITWHM is called the interval 2-tuple weighted harmonic mean (ITWHM) operator.

Based on the OWA and the ITWHM operators, Zhang [27] developed the interval 2-tuple ordered weighted harmonic mean (ITOWHM) operator as follows.
Definition 8. Let $\tilde{a}_{i}=\left[\left(s_{i}, \alpha_{i}\right),\left(t_{i}, \varepsilon_{i}\right)\right](i=1,2, \ldots, n)$ be a set of interval 2-tuples. An ITOWHM operator of dimension $n$ is a mapping ITOWHM: $\widetilde{S}^{n} \rightarrow \widetilde{S}$, which has an associated weight vector $\omega=\left(\omega_{1}, \omega_{2}, \ldots, \omega_{n}\right)^{T}$ with $\omega_{j} \in[0,1]$ and $\sum_{j=1}^{n} \omega_{j}=1$, such that

$$
\begin{aligned}
& \operatorname{ITOWhM}\left(\tilde{a}_{1}, \tilde{a}_{2}, \ldots, \tilde{a}_{n}\right)=\frac{1}{\bigoplus_{j=1}^{n}\left(\omega_{j} / \tilde{a}_{\sigma(j)}\right)} \\
& =\Delta\left[\frac{1}{\sum_{j=1}^{n} \omega_{j} /\left(\Delta^{-1}\left(s_{\sigma(j)}, \alpha_{\sigma(j)}\right)\right)},\right. \\
& \left.\frac{1}{\sum_{j=1}^{n} \omega_{j} /\left(\Delta^{-1}\left(t_{\sigma(j)}, \varepsilon_{\sigma(j)}\right)\right)}\right],
\end{aligned}
$$

where $\tilde{a}_{\sigma(j)}=\left[\left(s_{\sigma(j)}, \alpha_{\sigma(j)}\right),\left(t_{\sigma(j)}, \varepsilon_{\sigma(j)}\right)\right]$ is the $j$ th largest of $\tilde{a}_{i}(i=1,2, \ldots, n)$.

Obviously, the fundamental characteristic of the ITWHM operator is that it considers the importance of each given interval 2-tuple linguistic variable, whereas the fundamental characteristic of the ITOWHM operator is the reordering step, and it weights all the ordered positions of interval 2-tuple linguistic variables instead of weighting the given interval 2-tuples themselves. In the following, by combining the advantages of the ITWHM and ITOWHM operators, we develop an interval 2-tuple hybrid harmonic mean (ITHHM) operator that weights both the given interval 2-tuple linguistic variables and their ordered positions.

Definition 9. Let $\tilde{a}_{i}=\left[\left(s_{i}, \alpha_{i}\right),\left(t_{i}, \varepsilon_{i}\right)\right](i=1,2, \ldots, n)$ be a set of interval 2-tuples. An ITHHM operator of dimension $n$ is a mapping ITHHM: $\widetilde{S}^{n} \rightarrow \widetilde{S}$, which has an associated weight vector $\omega=\left(\omega_{1}, \omega_{2}, \ldots, \omega_{n}\right)^{T}$ with $\omega_{j} \in[0,1]$ and $\sum_{j=1}^{n} \omega_{j}=1$, such that

$$
\begin{gathered}
\operatorname{ITHhM}\left(\tilde{a}_{1}, \tilde{a}_{2}, \ldots, \tilde{a}_{n}\right)=\frac{1}{\bigoplus_{j=1}^{n}\left(\omega_{j} / \dot{\tilde{a}}_{\sigma(j)}\right)} \\
=\Delta\left[\frac{1}{\sum_{j=1}^{n} \omega_{j} /\left(\Delta^{-1}\left(\dot{s}_{\sigma(j)}, \dot{\alpha}_{\sigma(j)}\right)\right)},\right. \\
\left.\frac{1}{\sum_{j=1}^{n} \omega_{j} /\left(\Delta^{-1}\left(\dot{t}_{\sigma(j)}, \dot{\varepsilon}_{\sigma(j)}\right)\right)}\right],
\end{gathered}
$$

where $\dot{\tilde{a}}_{\sigma(j)}=\left[\left(\dot{s}_{\sigma(j)}, \dot{\alpha}_{\sigma(j)}\right),\left(\dot{t}_{\sigma(j)}, \dot{\varepsilon}_{\sigma(j)}\right)\right]$ is the $j$ th largest of the weighted interval 2-tuples $\dot{\tilde{a}}_{i}\left(\dot{\tilde{a}}_{i}=n w_{i} \widetilde{a}_{i}, i=1,2, \ldots, n\right)$, $w=\left(w_{1}, w_{2}, \ldots, w_{n}\right)^{T}$ is the weight vector of $\tilde{a}_{i}(i=$ $1,2, \ldots, n)$, with $w_{i} \in[0,1]$ and $\sum_{i=1}^{n} w_{i}=1$, and $n$ is the balancing coefficient.

In particular, if $w=(1 / n, 1 / n, \ldots, 1 / n)^{T}$, then the ITHHM operator is reduced to the ITOWHM operator; if $\omega=(1 / n, 1 / n, \ldots, 1 / n)^{T}$, then the ITHHM operator is reduced to the ITWHM operator. Thus, the ITHHM operator generalizes both the ITWHM and ITOWHM operators and 
reflects the importance degrees of both the given argument and its ordered position. Moreover, if the interval 2-tuples $\tilde{a}_{j}=\left[\left(s_{j}, \alpha_{j}\right),\left(t_{j}, \varepsilon_{j}\right)\right](j=1,2, \ldots, n)$ are degenerated to the 2 -tuples $\widehat{a}_{j}=\left(s_{j}, \alpha_{j}\right)(j=1,2, \ldots, n)$, then the ITHHM operator is reduced to the 2-tuple linguistic hybrid harmonic (2TLHH) operator [38].

To rank these interval 2-tuple linguistic arguments $\widetilde{a}_{i}(i=$ $1,2, \ldots, n)$, we first compare each argument $\tilde{a}_{i}$ with all arguments $\widetilde{a}_{j}(j=1,2, \ldots, n)$ by using (10) and let $p_{i j}=$ $p\left(\widetilde{a}_{i} \geq \tilde{a}_{j}\right)$. Then, we can construct a complementary matrix $P=\left(p_{i j}\right)_{n \times n}$, where $p_{i j} \geq 0, p_{i j}+p_{j i}=1$, and $p_{i i}=0.5$, $i, j=1,2, \ldots, n$. Summing all elements in each line of the matrix $P$, we have $p_{i}=\sum_{j=1}^{n} p_{i j}(i=1,2, \ldots, n)$. Then, we can rank the arguments $\widetilde{a}_{i}(i=1,2, \ldots, n)$ in descending order in accordance with the values of $p_{i}(i=1,2, \ldots, n)$.

Example 10. Assuming $S=\left\{s_{0}, s_{1}, \ldots, s_{6}\right\}$ is a linguistic term set, $\widetilde{a}_{1}=\left[\left(s_{3}, 0\right),\left(s_{4}, 0\right)\right], \widetilde{a}_{2}=\left[\left(s_{3}, 0\right),\left(s_{3}, 0\right)\right], \widetilde{a}_{3}=$ $\left[\left(s_{2}, 0\right),\left(s_{4}, 0\right)\right]$, and $\tilde{a}_{4}=\left[\left(s_{1}, 0\right),\left(s_{3}, 0\right)\right]$ and let $w=$ $(0.3,0.2,0.3,0.2)^{T}$ be the weight vector of $\widetilde{a}_{i}(i=1,2,3,4)$. Then, we get the weighted interval 2-tuples:

$$
\begin{aligned}
\dot{\tilde{a}}_{1} & =4 \times 0.3 \times\left[\left(s_{3}, 0\right),\left(s_{4}, 0\right)\right] \\
& =\left[\left(s_{4}, 0.133\right),\left(s_{5},-0.033\right)\right], \\
\dot{\tilde{a}}_{2} & =4 \times 0.2 \times\left[\left(s_{3}, 0\right),\left(s_{3}, 0\right)\right] \\
& =\left[\left(s_{2}, 0.067\right),\left(s_{2}, 0.067\right)\right], \\
\dot{\tilde{a}}_{3} & =4 \times 0.3 \times\left[\left(s_{2}, 0\right),\left(s_{4}, 0\right)\right] \\
& =\left[\left(s_{2}, 0.467\right),\left(s_{5},-0.033\right)\right], \\
\dot{\tilde{a}}_{4} & =4 \times 0.2 \times\left[\left(s_{1}, 0\right),\left(s_{3}, 0\right)\right] \\
& =\left[\left(s_{1}, 0.233\right),\left(s_{2}, 0.067\right)\right] .
\end{aligned}
$$

To rank these arguments, we first compare each argument $\dot{\tilde{a}}_{i}$ with all arguments $\dot{\tilde{a}}_{j}(j=1,2,3,4)$ by using (10) and then construct a complementary matrix

$$
P=\left[\begin{array}{cccc}
0.5 & 1 & 0.667 & 1 \\
1 & 0.5 & 0 & 1 \\
0.333 & 1 & 0.5 & 1 \\
0 & 0 & 0 & 0.5
\end{array}\right]
$$
have

Summing all elements in each line of the matrix $P$, we

$$
\begin{aligned}
& p_{1}=3.167, \\
& p_{2}=1.5, \\
& p_{3}=2.833, \\
& p_{4}=0.5 .
\end{aligned}
$$

Then, we rank the arguments $\dot{\tilde{a}}_{i}(i=1,2,3,4)$ in descending order in accordance with the values of $p_{i}(i=$ $1,2,3,4)$ :

$$
\begin{aligned}
& \dot{\tilde{a}}_{\sigma(1)}=\dot{\tilde{a}}_{1}=\left[\left(s_{4}, 0.133\right),\left(s_{5},-0.033\right)\right], \\
& \dot{\tilde{a}}_{\sigma(2)}=\dot{\tilde{a}}_{3}=\left[\left(s_{2}, 0.467\right),\left(s_{5},-0.033\right)\right], \\
& \dot{\tilde{a}}_{\sigma(3)}=\dot{\tilde{a}}_{2}=\left[\left(s_{2}, 0.067\right),\left(s_{2}, 0.067\right)\right], \\
& \dot{\tilde{a}}_{\sigma(4)}=\dot{\tilde{a}}_{4}=\left[\left(s_{1}, 0.233\right),\left(s_{2}, 0.067\right)\right] .
\end{aligned}
$$

Suppose that the weight vector of the ITHHM operator is $\omega=(0.2,0.3,0.3,0.2)^{T}$; then by (15), we get

$$
\begin{aligned}
& \operatorname{ITHHM}\left(\tilde{a}_{1}, \tilde{a}_{2}, \tilde{a}_{3}, \tilde{a}_{4}\right) \\
& \quad=\left(\frac{0.2}{\left[\left(s_{4}, 0.133\right),\left(s_{5},-0.033\right)\right]}\right. \\
& \quad \oplus \frac{0.3}{\left[\left(s_{2}, 0.467\right),\left(s_{5},-0.033\right)\right]} \\
& \quad \oplus \frac{0.3}{\left[\left(s_{2}, 0.067\right),\left(s_{2}, 0.067\right)\right]} \\
& \left.\quad \oplus \frac{0.2}{\left[\left(s_{1}, 0.233\right),\left(s_{2}, 0.067\right)\right]}\right)^{-1}=\Delta[0.300,0.533] \\
& \quad=\left[\left(s_{2},-0.033\right),\left(s_{3}, 0.033\right)\right] .
\end{aligned}
$$

\subsection{Induced Interval 2-Tuple Ordered Weighted Harmonic Mean Operator}

Definition 11. An induced ordered weighted harmonic mean (IOWHM) operator [39] is defined as follows:

$$
\begin{aligned}
& \operatorname{IOWHM}\left(\left\langle u_{1}, a_{1}\right\rangle,\left\langle u_{2}, a_{2}\right\rangle, \ldots,\left\langle u_{n}, a_{n}\right\rangle\right) \\
& =\frac{1}{\sum_{j=1}^{n} \omega_{j} / a_{\sigma(j)}},
\end{aligned}
$$

where $\omega=\left(\omega_{1}, \omega_{2}, \ldots, \omega_{n}\right)^{T}$ is a weight vector such that $\omega_{j} \epsilon$ $[0,1]$ and $\sum_{j=1}^{n} \omega_{j}=1, a_{\sigma(j)}$ is the $a_{i}$ value of the IOWA pair $\left\langle u_{i}, a_{i}\right\rangle$ having the $j$ th largest $u_{i}$, and $u_{i}$ in $\left\langle u_{i}, a_{i}\right\rangle$ is referred to as the order inducing variable.

In the following, we will develop an induced interval 2-tuple ordered weighted harmonic mean (I-ITOWHM) operator.

Definition 12. Let $\widetilde{a}_{i}=\left[\left(s_{i}, \alpha_{i}\right),\left(t_{i}, \varepsilon_{i}\right)\right](i=1,2, \ldots, n)$ be a set of interval 2-tuples and let $\omega=\left(\omega_{1}, \omega_{2}, \ldots, \omega_{n}\right)^{T}$ be an associated weight vector, with $\omega_{j} \in[0,1], \sum_{j=1}^{n} \omega_{j}=1$. The induced interval 2-tuple ordered weighted harmonic mean (I-ITOWHM) operator is defined as

$$
\begin{aligned}
& \operatorname{I-ITOWHM}\left(\left\langle u_{1}, \widetilde{a}_{1}\right\rangle,\left\langle u_{2}, \widetilde{a}_{2}\right\rangle, \ldots,\left\langle u_{n}, \widetilde{a}_{n}\right\rangle\right) \\
& \quad=\frac{1}{\bigoplus_{j=1}^{n}\left(\omega_{j} / \tilde{a}_{\sigma(j)}\right)}
\end{aligned}
$$




$$
\begin{aligned}
& =\Delta\left[\frac{1}{\sum_{j=1}^{n} \omega_{j} /\left(\Delta^{-1}\left(s_{\sigma(j)}, \alpha_{\sigma(j)}\right)\right)},\right. \\
& \left.\frac{1}{\sum_{j=1}^{n} \omega_{j} /\left(\Delta^{-1}\left(t_{\sigma(j)}, \varepsilon_{\sigma(j)}\right)\right)}\right],
\end{aligned}
$$

where $\tilde{a}_{\sigma(j)}=\left[\left(s_{\sigma(j)}, \alpha_{\sigma(j)}\right),\left(t_{\sigma(j)}, \varepsilon_{\sigma(j)}\right)\right]$ is the $\tilde{a}_{i}$ value of the IOWA pair $\left\langle u_{i}, \tilde{a}_{i}\right\rangle$ having the $j$ th largest $u_{i}$ and $u_{i}$ in $\left\langle u_{i}, \tilde{a}_{i}\right\rangle$ is referred to as the order inducing variable.

If there is a tie between $\left\langle u_{i}, \tilde{a}_{i}\right\rangle$ and $\left\langle u_{j}, \tilde{a}_{j}\right\rangle$ with respect to the order inducing variables such that $u_{i}=u_{j}$, in this case, we replace the argument component of each of $\left\langle u_{i}, \tilde{a}_{i}\right\rangle$ and $\left\langle u_{j}, \tilde{a}_{j}\right\rangle$ by their average $\left(\tilde{a}_{i}+\tilde{a}_{j}\right) / 2$ in the process of aggregation. If $k$ items are tied, then we replace these by $k$ replicas of their average. The weight vector $\omega=$ $\left(\omega_{1}, \omega_{2}, \ldots, \omega_{n}\right)^{T}$ can be determined by using some weight determining methods like the normal distribution based method [40].

In particular, if $\omega=(1 / n, 1 / n, \ldots, 1 / n)^{T}$, then the IITOWHM operator is reduced to the interval 2-tuple harmonic mean (ITHM) operator:

$$
\begin{aligned}
& \operatorname{ITHM}\left(\tilde{a}_{1}, \tilde{a}_{2}, \ldots, \tilde{a}_{n}\right)=\frac{n}{\bigoplus_{j=1}^{n}\left(1 / \tilde{a}_{j}\right)} \\
& =\Delta\left[\frac{n}{\sum_{j=1}^{n} 1 /\left(\Delta^{-1}\left(s_{j}, \alpha_{j}\right)\right)},\right. \\
& \left.\frac{n}{\sum_{j=1}^{n} 1 /\left(\Delta^{-1}\left(t_{j}, \varepsilon_{j}\right)\right)}\right] .
\end{aligned}
$$

If $u_{i}=i$, for all $i$, where $i$ is the ordered position of $\widetilde{a}_{i}$, the I-ITOWHM operator is reduced to the ITWHM operator; if $u_{i}=\tilde{a}_{i}$, for all $i$, then the I-ITOWHM operator is reduced to the ITOWHM operator. Moreover, if the interval 2-tuples $\tilde{a}_{i}=\left[\left(s_{i}, \alpha_{i}\right),\left(t_{i}, \varepsilon_{i}\right)\right](i=1,2, \ldots, n)$ are degenerated to the 2-tuples $\widehat{a}_{i}=\left(s_{i}, \alpha_{i}\right)(i=1,2, \ldots, n)$, then the I-ITOWHM operator is reduced to the induced 2-tuple ordered weighted harmonic mean (I-TOWHM) operator:

$$
\begin{aligned}
& \operatorname{I-ITOWHM}\left(\left\langle u_{1}, \hat{a}_{1}\right\rangle,\left\langle u_{2}, \widehat{a}_{2}\right\rangle, \ldots,\left\langle u_{n}, \widehat{a}_{n}\right\rangle\right) \\
& =\frac{1}{\bigoplus_{j=1}^{n}\left(\omega_{j} / \widehat{a}_{\sigma(j)}\right)} \\
& =\Delta\left[\frac{1}{\sum_{j=1}^{n} \omega_{j} /\left(\Delta^{-1}\left(s_{\sigma(j)}, \alpha_{\sigma(j)}\right)\right)}\right],
\end{aligned}
$$

where $\widehat{a}_{\sigma(j)}=\left(s_{\sigma(j)}, \alpha_{\sigma(j)}\right)$ is the $\widehat{a}_{i}$ value of the IOWA pair $\left\langle u_{i}, \widehat{a}_{i}\right\rangle$ having the $j$ th largest $u_{i}$, and $u_{i}$ in $\left\langle u_{i}, \widehat{a}_{i}\right\rangle$ is referred to as the order inducing variable. If there is a tie between $\left\langle u_{i}, \widehat{a}_{i}\right\rangle$ and $\left\langle u_{j}, \hat{a}_{j}\right\rangle$ with respect to the order inducing variables such that $u_{i}=u_{j}$, then we replace the argument component of each of $\left\langle u_{i}, \widehat{a}_{i}\right\rangle$ and $\left\langle u_{j}, \widehat{a}_{j}\right\rangle$ by their average $\left(\widehat{a}_{i}+\widehat{a}_{j}\right) / 2$ in the process of aggregation. If $k$ items are tied, then we replace these by $k$ replicas of their average.

The I-ITOWHM operator has the following properties similar to those of the IOWHM [39] and the IOWA operators [41].

Theorem 13 (commutativity). Consider

$$
\begin{aligned}
& \operatorname{I-ITOWHM}\left(\left\langle u_{1}, \tilde{a}_{1}\right\rangle,\left\langle u_{2}, \tilde{a}_{2}\right\rangle, \ldots,\left\langle u_{n}, \tilde{a}_{n}\right\rangle\right) \\
& \quad=\operatorname{I-ITOWHM}\left(\left\langle u_{1}, \tilde{a}_{1}^{\prime}\right\rangle,\left\langle u_{2}, \tilde{a}_{2}^{\prime}\right\rangle, \ldots,\left\langle u_{n}, \tilde{a}_{n}^{\prime}\right\rangle\right),
\end{aligned}
$$

where $\left(\left\langle u_{1}, \tilde{a}_{1}^{\prime}\right\rangle,\left\langle u_{2}, \tilde{a}_{2}^{\prime}\right\rangle, \ldots,\left\langle u_{n}, \tilde{a}_{n}^{\prime}\right\rangle\right)$ is any permutation of $\left(\left\langle u_{1}, \tilde{a}_{1}\right\rangle,\left\langle u_{2}, \widetilde{a}_{2}\right\rangle, \ldots,\left\langle u_{n}, \widetilde{a}_{n}\right\rangle\right)$.

Proof. Let

$$
\begin{aligned}
& \operatorname{I-ITOWHM}\left(\left\langle u_{1}, \tilde{a}_{1}\right\rangle,\left\langle u_{2}, \tilde{a}_{2}\right\rangle, \ldots,\left\langle u_{n}, \tilde{a}_{n}\right\rangle\right) \\
& \quad=\frac{1}{\bigoplus_{j=1}^{n}\left(\omega_{j} / \tilde{a}_{\sigma(j)}\right)}, \\
& \operatorname{I-ITOWHM}\left(\left\langle u_{1}, \tilde{a}_{1}^{\prime}\right\rangle,\left\langle u_{2}, \tilde{a}_{2}^{\prime}\right\rangle, \ldots,\left\langle u_{n}, \tilde{a}_{n}^{\prime}\right\rangle\right) \\
& =\frac{1}{\bigoplus_{j=1}^{n}\left(\omega_{j} / \tilde{a}_{\sigma(j)}^{\prime}\right)} .
\end{aligned}
$$

Since $\left(\left\langle u_{1}, \tilde{a}_{1}^{\prime}\right\rangle,\left\langle u_{2}, \tilde{a}_{2}^{\prime}\right\rangle, \ldots,\left\langle u_{n}, \tilde{a}_{n}^{\prime}\right\rangle\right)$ is any permutation of $\left(\left\langle u_{1}, \tilde{a}_{1}\right\rangle,\left\langle u_{2}, \tilde{a}_{2}\right\rangle, \ldots,\left\langle u_{n}, \tilde{a}_{n}\right\rangle\right)$, we have $\tilde{a}_{\sigma(j)}=\tilde{a}_{\sigma(j)}^{\prime}(j=$ $1,2, \ldots, n)$; then,

$$
\begin{aligned}
& \operatorname{I-ITOWHM}\left(\left\langle u_{1}, \tilde{a}_{1}\right\rangle,\left\langle u_{2}, \tilde{a}_{2}\right\rangle, \ldots,\left\langle u_{n}, \tilde{a}_{n}\right\rangle\right) \\
& \quad=\operatorname{I-ITOWHM}\left(\left\langle u_{1}, \tilde{a}_{1}^{\prime}\right\rangle,\left\langle u_{2}, \tilde{a}_{2}^{\prime}\right\rangle, \ldots,\left\langle u_{n}, \tilde{a}_{n}^{\prime}\right\rangle\right) .
\end{aligned}
$$

Theorem 14 (idempotency). If $\widetilde{a}_{i}=\tilde{a}$ for all $i$, then

$$
\operatorname{I-ITOWHM}\left(\left\langle u_{1}, \tilde{a}_{1}\right\rangle,\left\langle u_{2}, \tilde{a}_{2}\right\rangle, \ldots,\left\langle u_{n}, \tilde{a}_{n}\right\rangle\right)=\tilde{a} .
$$

Proof. Since $\widetilde{a}_{i}=\widetilde{a}$ for all $i$, we have

$$
\begin{gathered}
\operatorname{I-ITOWHM}\left(\left\langle u_{1}, \tilde{a}_{1}\right\rangle,\left\langle u_{2}, \tilde{a}_{2}\right\rangle, \ldots,\left\langle u_{n}, \tilde{a}_{n}\right\rangle\right) \\
=\frac{1}{\bigoplus_{j=1}^{n}\left(\omega_{j} / \widetilde{a}_{\sigma(j)}\right)}=\frac{1}{\sum_{j=1}^{n} \omega_{j} / \tilde{a}}=\tilde{a} .
\end{gathered}
$$

Theorem 15 (monotonicity). If $\widetilde{a}_{i} \leq \tilde{a}_{i}^{\prime}$ for all $i$, then

$$
\begin{aligned}
& \operatorname{I-ITOWHM}\left(\left\langle u_{1}, \tilde{a}_{1}\right\rangle,\left\langle u_{2}, \tilde{a}_{2}\right\rangle, \ldots,\left\langle u_{n}, \tilde{a}_{n}\right\rangle\right) \\
& \quad \leq \operatorname{I-ITOWHM}\left(\left\langle u_{1}, \tilde{a}_{1}^{\prime}\right\rangle,\left\langle u_{2}, \tilde{a}_{2}^{\prime}\right\rangle, \ldots,\left\langle u_{n}, \tilde{a}_{n}^{\prime}\right\rangle\right) .
\end{aligned}
$$

Proof. Let

$$
\begin{aligned}
& \operatorname{I-ITOWHM}\left(\left\langle u_{1}, \tilde{a}_{1}\right\rangle,\left\langle u_{2}, \tilde{a}_{2}\right\rangle, \ldots,\left\langle u_{n}, \tilde{a}_{n}\right\rangle\right) \\
& \quad=\frac{1}{\bigoplus_{j=1}^{n}\left(\omega_{j} / \tilde{a}_{\sigma(j)}\right)}, \\
& \operatorname{I-ITOWHM}\left(\left\langle u_{1}, \tilde{a}_{1}^{\prime}\right\rangle,\left\langle u_{2}, \tilde{a}_{2}^{\prime}\right\rangle, \ldots,\left\langle u_{n}, \tilde{a}_{n}^{\prime}\right\rangle\right)
\end{aligned}
$$

$$
=\frac{1}{\bigoplus_{j=1}^{n}\left(\omega_{j} / \tilde{a}_{\sigma(j)}^{\prime}\right)} .
$$


Since $\tilde{a}_{i} \leq \tilde{a}_{i}^{\prime}$ for all $i$, it follows that $\tilde{a}_{\sigma(j)} \leq \tilde{a}_{\sigma(j)}^{\prime}(j=$ $1,2, \ldots, n)$; then,

$$
\begin{aligned}
& \operatorname{I-ITOWHM}\left(\left\langle u_{1}, \tilde{a}_{1}\right\rangle,\left\langle u_{2}, \tilde{a}_{2}\right\rangle, \ldots,\left\langle u_{n}, \tilde{a}_{n}\right\rangle\right) \\
& \quad \leq \operatorname{I-ITOWHM}\left(\left\langle u_{1}, \tilde{a}_{1}^{\prime}\right\rangle,\left\langle u_{2}, \tilde{a}_{2}^{\prime}\right\rangle, \ldots,\left\langle u_{n}, \tilde{a}_{n}^{\prime}\right\rangle\right) .
\end{aligned}
$$

Example 16. Assuming $S=\left\{s_{0}, s_{1}, \ldots, s_{6}\right\}$ is a linguistic term set, $\left\langle u_{1}, \tilde{a}_{1}\right\rangle=\left\langle 0.2,\left[\left(s_{2}, 0\right),\left(s_{3}, 0\right)\right]\right\rangle,\left\langle u_{2}, \tilde{a}_{2}\right\rangle=\left\langle 0.4,\left[\left(s_{4}\right.\right.\right.$, $\left.\left.0),\left(s_{5}, 0\right)\right]\right\rangle,\left\langle u_{3}, \tilde{a}_{3}\right\rangle=\left\langle 0.3,\left[\left(s_{4}, 0\right),\left(s_{4}, 0\right)\right]\right\rangle$, and $\left\langle u_{4}, \tilde{a}_{4}\right\rangle=$ $\left\langle 0.8,\left[\left(s_{3}, 0\right),\left(s_{4}, 0\right)\right]\right\rangle$ are four IOWA pairs. Then, we desire to aggregate using the weight vector $\omega=(0.1,0.4,0.4,0.1)^{T}$. Performing the ordering of the IOWA pairs with respect to the first component, we get

$$
\begin{aligned}
& \left\langle u_{4}, \tilde{a}_{4}\right\rangle=\left\langle 0.8,\left[\left(s_{3}, 0\right),\left(s_{4}, 0\right)\right]\right\rangle, \\
& \left\langle u_{2}, \tilde{a}_{2}\right\rangle=\left\langle 0.4,\left[\left(s_{4}, 0\right),\left(s_{5}, 0\right)\right]\right\rangle, \\
& \left\langle u_{3}, \tilde{a}_{3}\right\rangle=\left\langle 0.3,\left[\left(s_{4}, 0\right),\left(s_{4}, 0\right)\right]\right\rangle, \\
& \left\langle u_{1}, \tilde{a}_{1}\right\rangle=\left\langle 0.2,\left[\left(s_{2}, 0\right),\left(s_{3}, 0\right)\right]\right\rangle .
\end{aligned}
$$

This ordering includes the ordered interval 2-tuple arguments:

$$
\begin{aligned}
& \tilde{a}_{\sigma(1)}=\tilde{a}_{4}=\left[\left(s_{3}, 0\right),\left(s_{4}, 0\right)\right], \\
& \tilde{a}_{\sigma(2)}=\widetilde{a}_{2}=\left[\left(s_{4}, 0\right),\left(s_{5}, 0\right)\right], \\
& \tilde{a}_{\sigma(3)}=\widetilde{a}_{3}=\left[\left(s_{4}, 0\right),\left(s_{4}, 0\right)\right], \\
& \tilde{a}_{\sigma(4)}=\tilde{a}_{1}=\left[\left(s_{2}, 0\right),\left(s_{3}, 0\right)\right] .
\end{aligned}
$$

Thus, we get an aggregated value

$$
\begin{aligned}
& \operatorname{I-ITOWHM}\left(\left\langle u_{1}, \tilde{a}_{1}\right\rangle,\left\langle u_{2}, \tilde{a}_{2}\right\rangle,\left\langle u_{3}, \tilde{a}_{3}\right\rangle,\left\langle u_{4}, \tilde{a}_{4}\right\rangle\right) \\
& \quad=\left(\frac{0.1}{\left[\left(s_{3}, 0\right),\left(s_{4}, 0\right)\right]} \oplus \frac{0.4}{\left[\left(s_{4}, 0\right),\left(s_{5}, 0\right)\right]}\right. \\
& \left.\quad \oplus \frac{0.4}{\left[\left(s_{4}, 0\right),\left(s_{4}, 0\right)\right]} \oplus \frac{0.1}{\left[\left(s_{2}, 0\right),\left(s_{3}, 0\right)\right]}\right)^{-1} \\
& =\Delta[0.588,0.699]=\left[\left(s_{4},-0.078\right),\left(s_{4}, 0.033\right)\right] .
\end{aligned}
$$

3.3. Induced Interval 2-Tuple Hybrid Harmonic Mean Operator. Inspired by the induced hybrid averaging (IHA) operator [42], in the following, we further generalize the ITHHM operator by using order inducing variables, obtaining the induced interval 2-tuple hybrid harmonic mean (I-ITHHM) operator.

Definition 17. Let $\tilde{a}_{i}=\left[\left(s_{i}, \alpha_{i}\right),\left(t_{i}, \varepsilon_{i}\right)\right](i=1,2, \ldots, n)$ be a set of interval 2-tuples. An I-ITHHM operator of dimension $n$ is a mapping I-ITHHM: $\widetilde{S}^{n} \rightarrow \widetilde{S}$, which has an associated weight vector $\omega=\left(\omega_{1}, \omega_{2}, \ldots, \omega_{n}\right)^{T}$ with $\omega_{j} \in[0,1]$ and $\sum_{j=1}^{n} \omega_{j}=1$, such that

$$
\begin{aligned}
& \operatorname{I-ITHHM}\left(\left\langle u_{1}, \tilde{a}_{1}\right\rangle,\left\langle u_{2}, \tilde{a}_{2}\right\rangle, \ldots,\left\langle u_{n}, \tilde{a}_{n}\right\rangle\right) \\
& =\frac{1}{\bigoplus_{j=1}^{n}\left(\omega_{j} / \dot{\tilde{a}}_{\sigma(j)}\right)} \\
& =\Delta\left[\frac{1}{\sum_{j=1}^{n} \omega_{j} /\left(\Delta^{-1}\left(\dot{s}_{\sigma(j)}, \dot{\alpha}_{\sigma(j)}\right)\right)},\right. \\
& \left.\frac{1}{\sum_{j=1}^{n} \omega_{j} /\left(\Delta^{-1}\left(\dot{t}_{\sigma(j)}, \dot{\varepsilon}_{\sigma(j)}\right)\right)}\right],
\end{aligned}
$$

where $\dot{\tilde{a}}_{\sigma(j)}=\left[\left(\dot{s}_{\sigma(j)}, \dot{\alpha}_{\sigma(j)}\right),\left(\dot{t}_{\sigma(j)}, \dot{\varepsilon}_{\sigma(j)}\right)\right]$ is the weighted interval 2-tuple $\dot{\tilde{a}}_{i}\left(\dot{\tilde{a}}_{i}=n w_{i} \widetilde{a}_{i}, i=1,2, \ldots, n\right)$ of the IOWA pair $\left\langle u_{i}, \tilde{a}_{i}\right\rangle$ having the $j$ th largest $u_{i}, u_{i}$ is the order inducing variable, $w=\left(w_{1}, w_{2}, \ldots, w_{n}\right)^{T}$ is the weight vector of $\widetilde{a}_{i}(i=$ $1,2, \ldots, n)$, with $w_{i} \in[0,1]$ and $\sum_{i=1}^{n} w_{i}=1$, and $n$ is the balancing coefficient.

In particular, if $w=(1 / n, 1 / n, \ldots, 1 / n)^{T}$, then the IITHHM operator is reduced to the I-ITOWHM operator; if $\omega=(1 / n, 1 / n, \ldots, 1 / n)^{T}$, then the I-ITHHM operator is reduced to the ITWHM operator. If $u_{i}=n w_{i} \widetilde{a}_{i}$, for all $i$, then the I-ITHHM operator is reduced to the ITHHM operator. Moreover, if the interval 2-tuples $\widetilde{a}_{i}=\left[\left(s_{i}, \alpha_{i}\right),\left(t_{i}, \varepsilon_{i}\right)\right](i=$ $1,2, \ldots, n)$ are degenerated to the 2-tuples $\widehat{a}_{i}=\left(s_{i}, \alpha_{i}\right)(i=$ $1,2, \ldots, n)$, then the I-ITHHM operator is reduced to the induced 2-tuple hybrid harmonic mean (I-THHM) operator:

$$
\begin{aligned}
\operatorname{I-THHM} & \left(\left\langle u_{1}, \hat{a}_{1}\right\rangle,\left\langle u_{2}, \hat{a}_{2}\right\rangle, \ldots,\left\langle u_{n}, \hat{a}_{n}\right\rangle\right) \\
& =\frac{1}{\bigoplus_{j=1}^{n}\left(\omega_{j} / \dot{\hat{a}}_{\sigma(j)}\right)} \\
& =\Delta\left[\frac{1}{\sum_{j=1}^{n} \omega_{j} /\left(\Delta^{-1}\left(\dot{s}_{\sigma(j)}, \dot{\alpha}_{\sigma(j)}\right)\right)}\right],
\end{aligned}
$$

where $\dot{\hat{a}}_{\sigma(j)}=\left(\dot{s}_{\sigma(j)}, \dot{\alpha}_{\sigma(j)}\right)$ is the weighted 2-tuple $\dot{\hat{a}}_{i}\left(\dot{\hat{a}}_{i}=\right.$ $\left.n w_{i} \widehat{a}_{i}, i=1,2, \ldots, n\right)$ of the IOWA pair $\left\langle u_{i}, \widehat{a}_{i}\right\rangle$ having the $j$ th largest $u_{i}, u_{i}$ is the order inducing variable, $w=$ $\left(w_{1}, w_{2}, \ldots, w_{n}\right)^{T}$ is the weight vector of $\hat{a}_{i}(i=1,2, \ldots, n)$, with $w_{i} \in[0,1]$ and $\sum_{i=1}^{n} w_{i}=1$, and $n$ is the balancing coefficient.

Example 18. Assuming $S=\left\{s_{0}, s_{1}, \ldots, s_{6}\right\}$ is a linguistic term set, $\left\langle u_{1}, \tilde{a}_{1}\right\rangle=\left\langle 0.2,\left[\left(s_{2}, 0\right),\left(s_{3}, 0\right)\right]\right\rangle,\left\langle u_{2}, \widetilde{a}_{2}\right\rangle=\left\langle 0.5,\left[\left(s_{1}, 0\right)\right.\right.$, $\left.\left.\left(s_{3}, 0\right)\right]\right\rangle,\left\langle u_{3}, \tilde{a}_{3}\right\rangle=\left\langle 0.3,\left[\left(s_{2}, 0\right),\left(s_{4}, 0\right)\right]\right\rangle$, and $\left\langle u_{4}, \tilde{a}_{4}\right\rangle=\langle 0.7$, $\left.\left[\left(s_{3}, 0\right),\left(s_{4}, 0\right)\right]\right\rangle$ are four IOWA pairs. Let $w=(0.2,0.3,0.2$, $0.3)^{T}$ be the weight vector of $\tilde{a}_{i}(i=1,2,3,4)$. Performing 
the ordering of the IOWA pairs with respect to the first component, we get

$$
\begin{aligned}
& \left\langle u_{4}, \tilde{a}_{4}\right\rangle=\left\langle 0.7,\left[\left(s_{3}, 0\right),\left(s_{4}, 0\right)\right]\right\rangle, \\
& \left\langle u_{2}, \tilde{a}_{2}\right\rangle=\left\langle 0.5,\left[\left(s_{1}, 0\right),\left(s_{3}, 0\right)\right]\right\rangle, \\
& \left\langle u_{3}, \tilde{a}_{3}\right\rangle=\left\langle 0.3,\left[\left(s_{2}, 0\right),\left(s_{4}, 0\right)\right]\right\rangle, \\
& \left\langle u_{1}, \tilde{a}_{1}\right\rangle=\left\langle 0.2,\left[\left(s_{2}, 0\right),\left(s_{3}, 0\right)\right]\right\rangle .
\end{aligned}
$$

Then, we get the weighted interval 2-tuples

$$
\begin{aligned}
\dot{\tilde{a}}_{\sigma(1)} & =4 \times 0.2 \times\left[\left(s_{3}, 0\right),\left(s_{4}, 0\right)\right] \\
& =\left[\left(s_{2}, 0.200\right),\left(s_{3}, 0.033\right)\right], \\
\dot{\tilde{a}}_{\sigma(2)} & =4 \times 0.3 \times\left[\left(s_{1}, 0\right),\left(s_{3}, 0\right)\right] \\
& =\left[\left(s_{1}, 0.043\right),\left(s_{4},-0.067\right)\right], \\
\dot{\tilde{a}}_{\sigma(3)} & =4 \times 0.2 \times\left[\left(s_{2}, 0\right),\left(s_{4}, 0\right)\right] \\
& =\left[\left(s_{2}, 0.200\right),\left(s_{3}, 0.033\right)\right], \\
\dot{\tilde{a}}_{\sigma(4)} & =4 \times 0.3 \times\left[\left(s_{2}, 0\right),\left(s_{3}, 0\right)\right] \\
& =\left[\left(s_{2}, 0.267\right),\left(s_{4},-0.067\right)\right] .
\end{aligned}
$$

Suppose that the weight vector of the I-ITHHM operator is $\omega=(0.2,0.4,0.3,0.1)^{T}$; then, by (36), we get

$$
\begin{aligned}
& \text { I-ITHHM }\left(\tilde{a}_{1}, \tilde{a}_{2}, \tilde{a}_{3}, \tilde{a}_{4}\right) \\
& =\left(\frac{0.2}{\left[\left(s_{2}, 0.200\right),\left(s_{3}, 0.033\right)\right]}\right. \\
& \oplus \frac{0.4}{\left[\left(s_{1}, 0.043\right),\left(s_{4},-0.067\right)\right]} \\
& \oplus \frac{0.3}{\left[\left(s_{2}, 0.200\right),\left(s_{3}, 0.033\right)\right]} \\
& \left.\oplus \frac{0.1}{\left[\left(s_{2}, 0.267\right),\left(s_{4},-0.067\right)\right]}\right)^{-1} \\
& =\Delta[0.258,0.565]=\left[\left(s_{2},-0.075\right),\left(s_{3}, 0.065\right)\right] .
\end{aligned}
$$

\section{An Approach to MAGDM with Interval 2-Tuple Linguistic Information}

In this section, we will develop an approach based on the proposed interval 2-tuple linguistic harmonic mean operators for the MAGDM problems in which both the attribute weights and the expert weights take the form of real numbers and the attribute values take the form of interval 2-tuple linguistic variables.

Suppose that a MAGDM problem has $l$ decision-makers $\mathrm{DM}_{k}(k=1,2, \ldots, l), m$ alternatives $A_{i}(i=1,2, \ldots, m)$, and $n$ decision attributes $C_{j}(j=1,2, \ldots, n)$. Each decisionmaker $\mathrm{DM}_{k}$ is given a weight $\lambda_{k}>0(k=1,2, \ldots, l)$ satisfying $\sum_{k=1}^{l} \lambda_{k}=1$ to reflect his/her relative importance in the group decision-making process. Let $D_{k}=\left(d_{i j}^{k}\right)_{m \times n}$ be the linguistic decision matrix of the $k$ th decision-maker, where $d_{i j}^{k}$ is the linguistic information provided by $\mathrm{DM}_{k}$ on the assessment of $A_{i}$ with respect to $C_{j}$. Let $w=\left(w_{1}, w_{2}, \ldots, w_{n}\right)^{T}$ be the weight vector of attributes, where $w_{j} \geq 0, j=$ $1,2, \ldots, n$, and $\sum_{j=1}^{n} w_{j}=1$. In addition, decision-makers may use different linguistic term sets to express their preference values.

In the following, we apply the I-ITHHM and the ITWHM operators to multiple attribute group decision-making with interval 2-tuple linguistic information.

Step 1. Convert the linguistic decision matrix $D_{k}=\left(d_{i j}^{k}\right)_{m \times n}$ into interval 2-tuple linguistic decision matrix $\widetilde{R}_{k}=$ $\left(\widetilde{r}_{i j}^{k}\right)_{m \times n}=\left(\left[\left(s_{i j}^{k}, 0\right),\left(t_{i j}^{k}, 0\right)\right]\right)_{m \times n}$, where $s_{i j}^{k}, t_{i j}^{k} \in S, S=\left\{s_{0}, s_{1}\right.$, $\left.\ldots, s_{g}\right\}$ and $s_{i j}^{k} \leq t_{i j}^{k}$.

Suppose that $\mathrm{DM}_{k}$ provides its assessments in a set of five linguistic terms and the linguistic term set is denoted as $S=\left\{s_{0}=\right.$ Very poor, $s_{1}=$ Poor, $s_{2}=$ Medium, $s_{3}=$ Good, $s_{4}=$ Very good $\}$. The linguistic information provided in the decision matrix $D_{k}$ can be converted into its corresponding interval 2-tuple linguistic assessments according to the following ways:

(i) A certain grade such as Poor, which can be written as $\left[\left(s_{1}, 0\right),\left(s_{1}, 0\right)\right]$;

(ii) an interval such as Poor-Medium, which means that the assessment of an alternative with respect to the attribute under consideration is between Poor and Medium which can be written as $\left[\left(s_{1}, 0\right),\left(s_{2}, 0\right)\right]$.

Step 2. Utilize the I-ITHHM operator which has an associated weight vector $\omega=\left(\omega_{1}, \omega_{2}, \ldots, \omega_{n}\right)^{T}$ with $\omega_{j} \in[0,1]$ and $\sum_{j=1}^{n} \omega_{j}=1$

$$
\begin{aligned}
\tilde{r}_{i j}= & {\left[\left(s_{i j}, \alpha_{i j}\right),\left(t_{i j}, \varepsilon_{i j}\right)\right] } \\
= & \operatorname{I-ITHHM}\left(\left\langle\lambda_{1}, \widetilde{r}_{i j}^{1}\right\rangle,\left\langle\lambda_{2}, \widetilde{r}_{i j}^{2}\right\rangle, \ldots,\left\langle\lambda_{n}, \widetilde{r}_{i j}^{l}\right\rangle\right) \\
= & \frac{1}{\bigoplus_{k=1}^{n}\left(\omega_{j} / \dot{\vec{r}}_{i j}^{\sigma(k)}\right)}, \\
& \quad i=1,2, \ldots, m, j=1,2, \ldots, n,
\end{aligned}
$$

to aggregate all the decision matrices $\widetilde{R}_{k}(k=1,2, \ldots, l)$ into a collective decision matrix $\widetilde{R}=\left(\widetilde{r}_{i j}\right)_{m \times n}$, where $\dot{\vec{r}}_{i j}^{\sigma(k)}$ is the weighted interval 2-tuple $\dot{\vec{r}}_{i j}^{k}\left(\dot{\tilde{r}}_{i j}^{k}=l \lambda_{k} \widetilde{r}_{i j}^{k}, k=1,2, \ldots, l\right)$ of the IOWA pair $\left\langle\lambda_{k}, \widetilde{r}_{i j}^{k}\right\rangle$ having the $k$ th largest $\lambda_{k}$.

Step 3. Utilize the decision information given in matrix $\widetilde{R}$ and the ITWHM operator

$$
\begin{aligned}
\tilde{r}_{i} & =\left[\left(s_{i}, \alpha_{i}\right),\left(t_{i}, \varepsilon_{i}\right)\right]=\operatorname{ITWHM}\left(\tilde{r}_{i 1}, \tilde{r}_{i 2}, \ldots, \tilde{r}_{i n}\right) \\
& =\frac{1}{\bigoplus_{j=1}^{n}\left(w_{j} / \widetilde{r}_{i j}\right)}, \quad i=1,2, \ldots, m,
\end{aligned}
$$


to derive the collective overall preference value $\tilde{r}_{i}$ of the alternative $A_{i}$.

Step 4. To rank these collective overall preference values $\widetilde{r}_{i}(i=1,2, \ldots, m)$, we first compare each $\widetilde{r}_{i}$ with all $\widetilde{r}_{j}(j=$ $1,2, \ldots, m)$ by using (10). Let $p_{i j}=p\left(\widetilde{r}_{i} \geq \widetilde{r}_{j}\right)$, and then we develop a complementary matrix as $P=\left(p_{i j}\right)_{m \times m}$, where $p_{i j} \geq 0, p_{i j}+p_{j i}=1$, and $p_{i i}=0.5, i, j=1,2, \ldots, m$.

Summing all elements in each line of the matrix $P$, we have $p_{i}=\sum_{j=1}^{m} p_{i j}, i=1,2, \ldots, m$. Then, we rank $\widetilde{r}_{i}(i=$ $1,2, \ldots, m)$ in descending order in accordance with the values of $p_{i}(i=1,2, \ldots, m)$.

Step 5. Rank all the alternatives $A_{i}(i=1,2, \ldots, m)$ and select the best one(s) in accordance with the collective overall preference values $\widetilde{r}_{i}(i=1,2, \ldots, m)$.

Step 6. End.

\section{An Illustrative Example}

In what follows, an illustrative example adapted from [43] is presented to illustrate the application of the developed method for material selection problem. This example problem is related with selection of a suitable work material for a product which needs to be designed for operating in a high-temperature environment. After preliminary screening, five alternative materials, $A_{i}(i=1,2, \ldots, 5)$, have been designated for further evaluation. An expert committee of four decision-makers, that is, $\mathrm{DM}_{1}, \mathrm{DM}_{2}, \mathrm{DM}_{3}$, and $\mathrm{DM}_{4}$, has been created in order to evaluate and select the most appropriate material for the application. The attributes which have been considered for the analysis are

$$
\begin{aligned}
& C_{1} \text { : tensile strength; } \\
& C_{2} \text { : Young's modulus; } \\
& C_{3} \text { : density; } \\
& C_{4} \text { : corrosion resistance. }
\end{aligned}
$$

The weight vector of these attributes is $w=(0.13,0.25$, $0.50,0.12)^{T}$. The four decision-makers, whose weight vector $\lambda=(0.15,0.20,0.35,0.30)^{T}$, employ different linguistic term sets to assess the suitability of the material with respect to the above selection attributes. Specifically, the decision-maker $\mathrm{DM}_{1}$ provides his assessments by using the linguistic term set $A ; \mathrm{DM}_{2}$ provides his assessments using $B ; \mathrm{DM}_{3}$ provides his assessments using $C ; \mathrm{DM}_{4}$ provides his assessments using $D$. These linguistic term sets are denoted as follows:

$$
\begin{aligned}
A & =\left\{a_{0}=\operatorname{Very} \operatorname{poor}(\mathrm{VP}), a_{1}=\operatorname{Poor}(\mathrm{P}), a_{2}\right. \\
& =\operatorname{Medium}(\mathrm{M}), a_{3}=\operatorname{Good}(\mathrm{G}), a_{4} \\
& =\operatorname{Very} \operatorname{good}(\mathrm{VG})\}, \\
B & =\left\{b_{0}=\operatorname{Very} \operatorname{poor}(\mathrm{VP}), b_{1}=\operatorname{Poor}(\mathrm{P}), b_{2}\right. \\
& =\operatorname{Medium} \operatorname{poor}(\mathrm{MP}), b_{3}=\operatorname{Medium}(\mathrm{M}), b_{4} \\
& =\operatorname{Medium} \operatorname{good}(\mathrm{MG}), b_{5}=\operatorname{Good}(\mathrm{G}), b_{6} \\
& =\operatorname{Very} \operatorname{good}(\mathrm{VG})\},
\end{aligned}
$$

$$
\begin{aligned}
C & =\left\{c_{0}=\operatorname{Extra} \text { poor }(\mathrm{EP}), c_{1}=\operatorname{Very} \text { poor }(\mathrm{VP}), c_{2}\right. \\
& =\operatorname{Poor}(\mathrm{P}), c_{3}=\operatorname{Medium} \operatorname{poor}(\mathrm{MP}), c_{4} \\
& =\operatorname{Medium}(\mathrm{M}), c_{5}=\operatorname{Medium} \operatorname{good}(\mathrm{MG}), c_{6} \\
& =\operatorname{Good}(\mathrm{G}), c_{7}=\operatorname{Very} \operatorname{good}(\mathrm{VG}), c_{8} \\
& =\operatorname{Extra} \operatorname{Good}(\mathrm{EG})\}, \\
D & =\left\{d_{0}=\operatorname{Very} \operatorname{poor}(\mathrm{VP}), d_{1}=\operatorname{Poor}(\mathrm{P}), d_{2}\right. \\
& =\operatorname{Medium}(\mathrm{M}), d_{3}=\operatorname{Good}(\mathrm{G}), d_{4} \\
& =\operatorname{Very} \operatorname{good}(\mathrm{VG})\} .
\end{aligned}
$$

The linguistic assessments of the five alternatives on each attribute provided by the four decision-makers are presented in Table 1.

Then, we utilize the method being proposed to get the most desirable alternative(s).

Step 1. Convert the linguistic decision matrix shown in Table 1 into interval 2-tuple linguistic decision matrix $\widetilde{R}_{k}=$ $\left(\left[\left(s_{i j}^{k}, 0\right),\left(t_{i j}^{k}, 0\right)\right]\right)_{5 \times 4}$, which is depicted in Table 2.

Step 2. Utilize the decision information given in matrixes $\widetilde{R}_{k}(k=1,2,3,4)$ and the I-ITHHM operator to get a collective decision matrix, as presented in Table 3.

In this example, the weight vector of the I-ITHHM operator is $\omega=(0.15,0.35,0.35,0.15)^{T}$ according to the normal distribution based method [40].

Step 3. Utilize the decision information given in matrix $\widetilde{R}$ and the ITWHM operator to obtain the collective overall preference value $\widetilde{r}_{i}$ of the alternative $A_{i}(i=1,2, \ldots, 5)$ :

$$
\begin{aligned}
& \tilde{r}_{1}=\Delta[0.811,0.865], \\
& \tilde{r}_{2}=\Delta[0.350,0.359], \\
& \tilde{r}_{3}=\Delta[0.317,0.365], \\
& \widetilde{r}_{4}=\Delta[0.686,0.716], \\
& \tilde{r}_{5}=\Delta[0.477,0.570] .
\end{aligned}
$$

Step 4. Calculate the values $p_{i}(i=1,2, \ldots, 5)$ of the collective overall preference values $r_{i}(i=1,2, \ldots, 5)$ by using $(10)$, and the results obtained are given as follows:

$$
\begin{aligned}
& p_{1}=4.5, \\
& p_{2}=1.239, \\
& p_{3}=0.761, \\
& p_{4}=3.5, \\
& p_{5}=2.5 .
\end{aligned}
$$

Step 5. Rank all the alternative $A_{i}(i=1,2, \ldots, 5)$ in accordance with the values of $p_{i}(i=1,2, \ldots, 5)$ in descending 
TABLE 1: Linguistic assessments of the five alternatives.

\begin{tabular}{|c|c|c|c|c|c|}
\hline \multirow{2}{*}{ Decision-makers } & \multirow{2}{*}{ Alternatives } & \multicolumn{4}{|c|}{ Attributes } \\
\hline & & $C_{1}$ & $\mathrm{C}_{2}$ & $C_{3}$ & $\mathrm{C}_{4}$ \\
\hline \multirow{5}{*}{$\mathrm{DM}_{1}$} & $A_{1}$ & G & G-VG & VG & G \\
\hline & $A_{2}$ & $M-G$ & M & G & M \\
\hline & $A_{3}$ & $\mathrm{M}$ & $\mathrm{M}$ & P-M & $\mathrm{P}$ \\
\hline & $A_{4}$ & G & G-VG & M & G \\
\hline & $A_{5}$ & M & $M-G$ & G & G \\
\hline \multirow{5}{*}{$\mathrm{DM}_{2}$} & $A_{1}$ & VG & VG & G & VG \\
\hline & $A_{2}$ & MP & $\mathrm{M}$ & $\mathrm{P}$ & $\mathrm{M}$ \\
\hline & $A_{3}$ & MP & MG & M & MP \\
\hline & $A_{4}$ & G & G & G & MG-G \\
\hline & $A_{5}$ & $M-G$ & M & MP-M & MG \\
\hline \multirow{5}{*}{$\mathrm{DM}_{3}$} & $A_{1}$ & EG & VG & VG-EG & VG \\
\hline & $A_{2}$ & M-MG & $M-G$ & G & G \\
\hline & $A_{3}$ & $\mathrm{P}$ & $\mathrm{MP}$ & $\mathrm{P}$ & P-MP \\
\hline & $A_{4}$ & VG & VG & G-VG & G-VG \\
\hline & $A_{5}$ & M & $\mathrm{M}$ & G-VG & G \\
\hline \multirow{5}{*}{$\mathrm{DM}_{4}$} & $A_{1}$ & VG & VG & G-VG & $\mathrm{VG}$ \\
\hline & $A_{2}$ & M-G & $\mathrm{M}$ & G & G \\
\hline & $A_{3}$ & $\mathrm{P}-\mathrm{M}$ & M & $\mathrm{P}$ & $\mathrm{P}-\mathrm{M}$ \\
\hline & $A_{4}$ & G & VG & G & G-VG \\
\hline & $A_{5}$ & M-G & M & G & G \\
\hline
\end{tabular}

TABLE 2: Interval 2-tuple linguistic decision matrix of the four decision makers.

\begin{tabular}{|c|c|c|c|c|c|}
\hline \multirow{2}{*}{ Decision-makers } & \multirow{2}{*}{ Alternatives } & \multicolumn{4}{|c|}{ Attributes } \\
\hline & & $C_{1}$ & $C_{2}$ & $C_{3}$ & $C_{4}$ \\
\hline \multirow{5}{*}{$\mathrm{DM}_{1}$} & $A_{1}$ & {$\left[\left(a_{3}, 0\right),\left(a_{3}, 0\right)\right]$} & {$\left[\left(a_{3}, 0\right),\left(a_{4}, 0\right)\right]$} & {$\left[\left(a_{4}, 0\right),\left(a_{4}, 0\right)\right]$} & {$\left[\left(a_{3}, 0\right),\left(a_{3}, 0\right)\right]$} \\
\hline & $A_{2}$ & {$\left[\left(a_{2}, 0\right),\left(a_{3}, 0\right)\right]$} & {$\left[\left(a_{2}, 0\right),\left(a_{2}, 0\right)\right]$} & {$\left[\left(a_{3}, 0\right),\left(a_{3}, 0\right)\right]$} & {$\left[\left(a_{2}, 0\right),\left(a_{2}, 0\right)\right]$} \\
\hline & $A_{3}$ & {$\left[\left(a_{2}, 0\right),\left(a_{2}, 0\right)\right]$} & {$\left[\left(a_{2}, 0\right),\left(a_{2}, 0\right)\right]$} & {$\left[\left(a_{1}, 0\right),\left(a_{2}, 0\right)\right]$} & {$\left[\left(a_{1}, 0\right),\left(a_{1}, 0\right)\right]$} \\
\hline & $A_{4}$ & {$\left[\left(a_{3}, 0\right),\left(a_{3}, 0\right)\right]$} & {$\left[\left(a_{3}, 0\right),\left(a_{4}, 0\right)\right]$} & {$\left[\left(a_{2}, 0\right),\left(a_{2}, 0\right)\right]$} & {$\left[\left(a_{3}, 0\right),\left(a_{3}, 0\right)\right]$} \\
\hline & $A_{5}$ & {$\left[\left(a_{2}, 0\right),\left(a_{2}, 0\right)\right]$} & {$\left[\left(a_{2}, 0\right),\left(a_{3}, 0\right)\right]$} & {$\left[\left(a_{3}, 0\right),\left(a_{3}, 0\right)\right]$} & {$\left[\left(a_{3}, 0\right),\left(a_{3}, 0\right)\right]$} \\
\hline \multirow{5}{*}{$\mathrm{DM}_{2}$} & $A_{1}$ & {$\left[\left(b_{6}, 0\right),\left(b_{6}, 0\right)\right]$} & {$\left[\left(b_{6}, 0\right),\left(b_{6}, 0\right)\right]$} & {$\left[\left(b_{5}, 0\right),\left(b_{5}, 0\right)\right]$} & {$\left[\left(b_{6}, 0\right),\left(b_{6}, 0\right)\right]$} \\
\hline & $A_{2}$ & {$\left[\left(b_{2}, 0\right),\left(b_{2}, 0\right)\right]$} & {$\left[\left(b_{3}, 0\right),\left(b_{3}, 0\right)\right]$} & {$\left[\left(b_{1}, 0\right),\left(b_{1}, 0\right)\right]$} & {$\left[\left(b_{3}, 0\right),\left(b_{3}, 0\right)\right]$} \\
\hline & $A_{3}$ & {$\left[\left(b_{2}, 0\right),\left(b_{2}, 0\right)\right]$} & {$\left[\left(b_{4}, 0\right),\left(b_{4}, 0\right)\right]$} & {$\left[\left(b_{3}, 0\right),\left(b_{3}, 0\right)\right]$} & {$\left[\left(b_{2}, 0\right),\left(b_{2}, 0\right)\right]$} \\
\hline & $A_{4}$ & {$\left[\left(b_{5}, 0\right),\left(b_{5}, 0\right)\right]$} & {$\left[\left(b_{5}, 0\right),\left(b_{5}, 0\right)\right]$} & {$\left[\left(b_{5}, 0\right),\left(b_{5}, 0\right)\right]$} & {$\left[\left(b_{4}, 0\right),\left(b_{5}, 0\right)\right]$} \\
\hline & $A_{5}$ & {$\left[\left(b_{3}, 0\right),\left(b_{5}, 0\right)\right]$} & {$\left[\left(b_{3}, 0\right),\left(b_{3}, 0\right)\right]$} & {$\left[\left(b_{2}, 0\right),\left(b_{3}, 0\right)\right]$} & {$\left[\left(b_{4}, 0\right),\left(b_{4}, 0\right)\right]$} \\
\hline \multirow{5}{*}{$\mathrm{DM}_{3}$} & $A_{1}$ & {$\left[\left(c_{8}, 0\right),\left(c_{8}, 0\right)\right]$} & {$\left[\left(c_{7}, 0\right),\left(c_{7}, 0\right)\right]$} & {$\left[\left(c_{7}, 0\right),\left(c_{8}, 0\right)\right]$} & {$\left[\left(c_{7}, 0\right),\left(c_{7}, 0\right)\right]$} \\
\hline & $A_{2}$ & {$\left[\left(c_{4}, 0\right),\left(c_{5}, 0\right)\right]$} & {$\left[\left(c_{4}, 0\right),\left(c_{6}, 0\right)\right]$} & {$\left[\left(c_{6}, 0\right),\left(c_{6}, 0\right)\right]$} & {$\left[\left(c_{6}, 0\right),\left(c_{6}, 0\right)\right]$} \\
\hline & $A_{3}$ & {$\left[\left(c_{2}, 0\right),\left(c_{2}, 0\right)\right]$} & {$\left[\left(c_{3}, 0\right),\left(c_{3}, 0\right)\right]$} & {$\left[\left(c_{2}, 0\right),\left(c_{2}, 0\right)\right]$} & {$\left[\left(c_{2}, 0\right),\left(c_{3}, 0\right)\right]$} \\
\hline & $A_{4}$ & {$\left[\left(c_{7}, 0\right),\left(c_{7}, 0\right)\right]$} & {$\left[\left(c_{7}, 0\right),\left(c_{7}, 0\right)\right]$} & {$\left[\left(c_{6}, 0\right),\left(c_{7}, 0\right)\right]$} & {$\left[\left(c_{6}, 0\right),\left(c_{7}, 0\right)\right]$} \\
\hline & $A_{5}$ & {$\left[\left(c_{4}, 0\right),\left(c_{4}, 0\right)\right]$} & {$\left[\left(c_{4}, 0\right),\left(c_{4}, 0\right)\right]$} & {$\left[\left(c_{6}, 0\right),\left(c_{7}, 0\right)\right]$} & {$\left[\left(c_{6}, 0\right),\left(c_{6}, 0\right)\right]$} \\
\hline \multirow{5}{*}{$\mathrm{DM}_{4}$} & $A_{1}$ & {$\left[\left(d_{4}, 0\right),\left(d_{4}, 0\right)\right]$} & {$\left[\left(d_{4}, 0\right),\left(d_{4}, 0\right)\right]$} & {$\left[\left(d_{3}, 0\right),\left(d_{4}, 0\right)\right]$} & {$\left[\left(d_{4}, 0\right),\left(d_{4}, 0\right)\right]$} \\
\hline & $A_{2}$ & {$\left[\left(d_{2}, 0\right),\left(d_{3}, 0\right)\right]$} & {$\left[\left(d_{2}, 0\right),\left(d_{2}, 0\right)\right]$} & {$\left[\left(d_{3}, 0\right),\left(d_{3}, 0\right)\right]$} & {$\left[\left(d_{3}, 0\right),\left(d_{3}, 0\right)\right]$} \\
\hline & $A_{3}$ & {$\left[\left(d_{1}, 0\right),\left(d_{2}, 0\right)\right]$} & {$\left[\left(d_{2}, 0\right),\left(d_{2}, 0\right)\right]$} & {$\left[\left(d_{1}, 0\right),\left(d_{1}, 0\right)\right]$} & {$\left[\left(d_{1}, 0\right),\left(d_{2}, 0\right)\right]$} \\
\hline & $A_{4}$ & {$\left[\left(d_{3}, 0\right),\left(d_{3}, 0\right)\right]$} & {$\left[\left(d_{4}, 0\right),\left(d_{4}, 0\right)\right]$} & {$\left[\left(d_{3}, 0\right),\left(d_{3}, 0\right)\right]$} & {$\left[\left(d_{3}, 0\right),\left(d_{4}, 0\right)\right]$} \\
\hline & $A_{5}$ & {$\left[\left(d_{2}, 0\right),\left(d_{3}, 0\right)\right]$} & {$\left[\left(d_{2}, 0\right),\left(d_{2}, 0\right)\right]$} & {$\left[\left(d_{3}, 0\right),\left(d_{3}, 0\right)\right]$} & {$\left[\left(d_{3}, 0\right),\left(d_{3}, 0\right)\right]$} \\
\hline
\end{tabular}


TABLE 3: Collective decision matrix by I-ITHHM.

\begin{tabular}{lcccc}
\hline Alternatives & $C_{1}$ & \multicolumn{3}{c}{ Attributes } \\
\\
\hline$A_{1}$ & $\Delta[0.855,0.855]$ & $C_{2}$ & $C_{3}$ & $\Delta[0.844,0.844]$ \\
$A_{2}$ & $\Delta[0.383,0.453]$ & $\Delta[0.844,0.908]$ & $\Delta[0.777,0.852]$ & $\Delta[0.524,0.524]$ \\
$A_{3}$ & $\Delta[0.293,0.354]$ & $\Delta[0.460,0.476]$ & $\Delta[0.287,0.287]$ & $\Delta[0.256,0.314]$ \\
$A_{4}$ & $\Delta[0.730,0.730]$ & $\Delta[0.786,0.841]$ & $\Delta[0.288,0.337]$ & $\Delta[0.657,0.786]$ \\
$A_{5}$ & $\Delta[0.460,0.614]$ & $\Delta[0.460,0.499]$ & $\Delta[0.642,0.651]$ & $\Delta[0.657,0.657]$ \\
\hline
\end{tabular}

TABLE 4: Ranking comparisons.

\begin{tabular}{llr}
\hline Operators & \multicolumn{1}{c}{ Ranking } & The best alternative \\
\hline By ITHHM & $A_{1}>A_{4}>A_{5}>A_{2}>A_{3}$ & $A_{1}$ \\
By I-ITOWHM & $A_{1}>A_{4}>A_{5}>A_{2}>A_{3}$ & $A_{1}$ \\
By I-ITHHM & $A_{1}>A_{4}>A_{5}>A_{2}>A_{3}$ & $A_{1}$ \\
By FWHM + FHHM & $A_{1}>A_{4}>A_{5}>A_{2}>A_{3}$ & $A_{1}$ \\
By ULHHM + ULWHM & $A_{1}>A_{4}>A_{5}>A_{2}>A_{3}$ & $A_{1}$ \\
By THWA + TWA & $A_{1}>A_{4}>A_{5}>A_{2}>A_{3}$ & $A_{1}$ \\
\hline
\end{tabular}

order: $A_{1}>A_{4}>A_{5}>A_{2}>A_{3}$, and thus the most desirable alternative is $A_{1}$.

Next, we use the ITHHM and the I-ITOWHM operators in Step 2 to rank the alternatives. The ranking results are shown in Table 4 . It is easy to see from Table 4 that the best selection is the alternative $A_{1}$ for all the aggregation operators used in this example. It should be noted that, depending on the aggregation operators used, the ranking orders of the alternative materials may be different. Therefore, according to the particular type of aggregation operator used, the results may lead to different decisions. But, in this example, it seems clear that $A_{1}$ is the optimal choice. Hence, the committee can recommend that material $A_{1}$ is the most suitable selection for the product.

In addition, to further evaluate the proposed method, we use the above material selection problem to analyze some comparable methods, which are based on the aggregation operators proposed by $\mathrm{Xu}$ [30], Park et al. [31], and Wan [15], respectively. In the first method, the fuzzy hybrid harmonic mean (FHHM) operator is utilized in the aggregation stage and the fuzzy weighted harmonic mean (FWHM) operator is used in the exploitation stage. Similarly, the second method employs the uncertain linguistic hybrid harmonic mean (ULHHM) operator in the aggregation stage and the uncertain linguistic weighted harmonic mean (ULWHM) operator in the exploitation stage; the third method employs the 2tuple hybrid weighted arithmetic average (THWA) operator in the aggregation stage and the 2-tuple weighted averaging (TWA) operator in the exploitation stage. The ranking results of the four alternatives derived by using these methods are presented in Table 4.

According to Table 4, the ranking orders of the alternatives obtained by the method proposed in this paper are exactly the same as those determined by the methods based on previous operators. Thus, the proposed method is validated. However, in comparison with the listed methods, the proposed approach using interval 2-tuple linguistic harmonic mean operators is more reasonable and flexible for solving MAGDM problems because of the following:

(i) It has exact characteristic in linguistic information processing and can effectively avoid the loss and distortion of information which occur formerly in other types of linguistic computational models.

(ii) The uncertainty and diversity of decision-makers' assessment information can be well reflected and modelled using interval 2-tuple linguistic variables. Moreover, the linguistic term sets with different granularity of uncertainty can be used by decision-makers for assessing alternatives.

(iii) We can represent more complex group decisionmaking processes that include psychological factors such as time pressure and personal affects to each alternative, by using order inducing variables in the aggregation stage.

\section{Conclusions}

In this paper, we have developed some new harmonic aggregation operators including the interval 2-tuple hybrid harmonic mean (ITHHM) operator, the induced interval 2-tuple ordered weighted harmonic mean (I-ITOWHM) operator, and the induced interval 2-tuple hybrid harmonic mean (I-ITHHM) operator. It has been shown that both the ITWHM and ITOWHM operators are the special cases of the ITHHM operator, and if all the input interval 2-tuple data are reduced to the 2-tuple data, then the developed operators are reduced to the 2TLHH operator, the I-TOWHM operator, and the I-THHM operator, respectively. We have studied some desired properties of the I-ITOWHM operator, such as commutativity, idempotency, and monotonicity, and applied the I-ITHHM and the ITWHM operators to multiple attribute group decision-making with interval 2-tuple 
linguistic information. Finally, a material selection example has been given to verify the developed method and to demonstrate its practicality and effectiveness.

In the future, we expect to present further extensions to the proposed approach by adding new characteristics in the decision process and consider the potential applications of the developed interval 2-tuple linguistic harmonic mean operators to other fields. First, in many real-world situations, decision-makers may hesitate among several possible linguistic values or think of richer expressions for assessing an alternative because of uncertainty. Thus, extending the proposed decision model by using the hesitant fuzzy linguistic term sets [44-46] is recommended in future research to solve the material selection problem more efficiently. Second, the proposed approach for group decision-making based on interval 2-tuple linguistic harmonic mean operators is a general method, which can be easily applied to deal with other decision-making problems such as robot evaluation and selection, green supply chain management, and electric vehicle charging station planning.

\section{Competing Interests}

The authors declare no competing interests.

\section{Acknowledgments}

This work was partially supported by the National Natural Science Foundation of China (no. 71402090), the National Social Science Foundation (no. 15CGL003), the Program for Professor of Special Appointment (Young Eastern Scholar) at Shanghai Institutions of Higher Learning (no. QD2015019), and the Shanghai Science and Technology Innovation Action Plan Soft Science Foundation (no. 16692103800).

\section{References}

[1] F. Herrera and L. Martínez, "A 2-tuple fuzzy linguistic representation model for computing with words," IEEE Transactions on Fuzzy Systems, vol. 8, no. 6, pp. 746-752, 2000.

[2] Z. Chen, P. Liu, and Z. Pei, "An approach to multiple attribute group decision making based on linguistic intuitionistic fuzzy numbers," International Journal of Computational Intelligence Systems, vol. 8, no. 4, pp. 747-760, 2015.

[3] L. Martínez, R. M. Rodriguez, and F. Herrera, The 2-Tuple Linguistic Model: Computing with Words in Decision Making, Springer, Berlin, Germany, 2015.

[4] L. Martínez and F. Herrera, "An overview on the 2-tuple linguistic model for computing with words in decision making: extensions, applications and challenges," Information Sciences, vol. 207, pp. 1-18, 2012.

[5] R. M. Rodríguez and L. Martínez, "An analysis of symbolic linguistic computing models in decision making," International Journal of General Systems, vol. 42, no. 1, pp. 121-136, 2013.

[6] Y.-X. Xue, J.-X. You, X. Zhao, and H.-C. Liu, "An integrated linguistic MCDM approach for robot evaluation and selection with incomplete weight information," International Journal of Production Research, vol. 54, no. 18, pp. 5452-5467, 2016.

[7] Z. Hu, C. Rao, Y. Zheng, and D. Huang, "Optimization decision of supplier selection in green procurement under the mode of low carbon economy," International Journal of Computational Intelligence Systems, vol. 8, no. 3, pp. 407-421, 2015.

[8] J. M. Merigó, M. Casanovas, and L. Martínez, "Linguistic aggregation operators for linguistic decision making based on the Dempster-Shafer theory of evidence," International Journal of Uncertainty, Fuzziness and Knowledge-Based Systems, vol. 18, no. 3, pp. 287-304, 2010.

[9] C. Lu, J.-X. You, H.-C. Liu, and P. Li, "Health-care waste treatment technology selection using the interval 2-tuple induced TOPSIS method," International Journal of Environmental Research and Public Health, vol. 13, no. 6, p. 562, 2016.

[10] H. C. Liu, J. X. You, P. Li, and Q. Su, "Failure mode and effect analysis under uncertainty: an integrated multiple criteria decision making approach," IEEE Transactions on Reliability, 2016.

[11] G.-W. Wei, "A method for multiple attribute group decision making based on the ET-WG and ET-OWG operators with 2tuple linguistic information," Expert Systems with Applications, vol. 37, no. 12, pp. 7895-7900, 2010.

[12] G.-W. Wei, "Some generalized aggregating operators with linguistic information and their application to multiple attribute group decision making," Computers and Industrial Engineering, vol. 61, no. 1, pp. 32-38, 2011.

[13] W. Yang and Z. Chen, "New aggregation operators based on the Choquet integral and 2-tuple linguistic information," Expert Systems with Applications, vol. 39, no. 3, pp. 2662-2668, 2012.

[14] Y. Xu and H. Wang, "Approaches based on 2-tuple linguistic power aggregation operators for multiple attribute group decision making under linguistic environment," Applied Soft Computing Journal, vol. 11, no. 5, pp. 3988-3997, 2011.

[15] S.-P. Wan, "2-Tuple linguistic hybrid arithmetic aggregation operators and application to multi-attribute group decision making," Knowledge-Based Systems, vol. 45, pp. 31-40, 2013.

[16] H. Sonia, H. Nesrin, and H. Chabouchoub, "Development of some linguistic aggregation operators with conservation of interaction between criteria and their application in multiple attribute group decision problems," TOP, 2016.

[17] J. Lin, Q. Zhang, and F. Meng, "An approach for facility location selection based on optimal aggregation operator," KnowledgeBased Systems, vol. 85, pp. 143-158, 2015.

[18] Y. Li and P. Liu, "Some Heronian mean operators with 2tuple linguistic information and their application to multiple attribute group decision making," Technological and Economic Development of Economy, vol. 21, no. 5, pp. 797-814, 2015.

[19] Y. Ju, X. Liu, and A. Wang, "Some new Shapley 2-tuple linguistic Choquet aggregation operators and their applications to multiple attribute group decision making," Soft Computing, pp. 1-17, 2015.

[20] L. Jiang, H. Liu, and J. Cai, "The power average operator for unbalanced linguistic term sets," Information Fusion, vol. 22, pp. 85-94, 2015.

[21] B. Dutta, D. Guha, and R. Mesiar, "A model based on linguistic 2-tuples for dealing with heterogeneous relationship among attributes in multi-expert decision making," IEEE Transactions on Fuzzy Systems, vol. 23, no. 5, pp. 1817-1831, 2015.

[22] B. Dutta and D. Guha, "Partitioned Bonferroni mean based on linguistic 2-tuple for dealing with multi-attribute group decision making," Applied Soft Computing Journal, vol. 37, pp. 166-179, 2015. 
[23] J.-Q. Wang, D.-D. Wang, H.-Y. Zhang, and X.-H. Chen, "Multicriteria group decision making method based on interval 2tuple linguistic information and Choquet integral aggregation operators," Soft Computing, vol. 19, no. 2, pp. 389-405, 2015.

[24] X.-Y. You, J.-X. You, H.-C. Liu, and L. Zhen, "Group multicriteria supplier selection using an extended VIKOR method with interval 2-tuple linguistic information," Expert Systems with Applications, vol. 42, no. 4, pp. 1906-1916, 2015.

[25] H.-C. Liu, P. Li, J.-X. You, and Y.-Z. Chen, "A novel approach for FMEA: combination of interval 2-tuple linguistic variables and gray relational analysis," Quality and Reliability Engineering International, vol. 31, no. 5, pp. 761-772, 2015.

[26] H. Zhang, "The multiattribute group decision making method based on aggregation operators with interval-valued 2-tuple linguistic information," Mathematical and Computer Modelling, vol. 56, no. 1-2, pp. 27-35, 2012.

[27] H. Zhang, "Some interval-valued 2-tuple linguistic aggregation operators and application in multiattribute group decision making," Applied Mathematical Modelling, vol. 37, no. 6, pp. 4269-4282, 2013.

[28] X. Liu, Z. Tao, H. Chen, and L. Zhou, "A new interval-valued 2-tuple linguistic Bonferroni mean operator and its application to multiattribute group decision making," International Journal of Fuzzy Systems, 2016.

[29] Q. Wu, P. Wu, Y. Zhou, L. Zhou, H. Chen, and X. Ma, "Some 2-tuple linguistic generalized power aggregation operators and their applications to multiple attribute group decision making," Journal of Intelligent and Fuzzy Systems, vol. 29, no. 1, pp. 423436, 2015.

[30] Z. Xu, "Fuzzy harmonic mean operators," International Journal of Intelligent Systems, vol. 24, no. 2, pp. 152-172, 2009.

[31] J. H. Park, M. G. Gwak, and Y. C. Kwun, "Uncertain linguistic harmonic mean operators and their applications to multiple attribute group decision making," Computing, vol. 93, no. 1, pp. 47-64, 2011.

[32] L. A. Zadeh, "The concept of a linguistic variable and its application to approximate reasoning-I," Information Sciences, vol. 8, no. 3, pp. 199-249, 1975.

[33] W.-S. Tai and C.-T. Chen, "A new evaluation model for intellectual capital based on computing with linguistic variable," Expert Systems with Applications, vol. 36, no. 2, pp. 3483-3488, 2009.

[34] H.-C. Liu, Q.-L. Lin, and J. Wu, "Dependent interval 2tuple linguistic aggregation operators and their application to multiple attribute group decision making," International Journal of Uncertainty, Fuzziness and Knowledge-Based Systems, vol. 22, no. 5, pp. 717-735, 2014.

[35] Z. Xu, "Uncertain linguistic aggregation operators based approach to multiple attribute group decision making under uncertain linguistic environment," Information Sciences, vol. 168, no. 1-4, pp. 171-184, 2004.

[36] Z. S. Xu, "An approach based on the uncertain LOWG and induced uncertain LOWG operators to group decision making with uncertain multiplicative linguistic preference relations," Decision Support Systems, vol. 41, no. 2, pp. 488-499, 2006.

[37] P. S. Bullen, D. S. Mitrinovi, and P. M. Vasi, Means and Their Inequalities, Reidel, Dordrecht, The Netherlands, 1988.

[38] J. H. Park, J. M. Park, and Y. C. Kwun, "2-Tuple linguistic harmonic operators and their applications in group decision making," Knowledge-Based Systems, vol. 44, pp. 10-19, 2013.
[39] H. Y. Chen, C. L. Liu, and Z. H. Sheng, "Induced ordered weighted harmonic averaging (IOWHA) operator and its application to combination forecasting method," Chinese Journal of Management Science, vol. 12, no. 5, pp. 35-40, 2004.

[40] Z. S. Xu, "An overview of methods for determining OWA weights," International Journal of Intelligent Systems, vol. 20, no. 8, pp. 843-865, 2005.

[41] R. R. Yager and D. P. Filev, "Induced ordered weighted averaging operators," IEEE Transactions on Systems, Man, and Cybernetics, Part B: Cybernetics, vol. 29, no. 2, pp. 141-150, 1999.

[42] J. M. Merigó and M. C. Ramón, "The induced generalized hybrid averaging operator and its application in financial decision making," International Journal of Human and Social Science, vol. 4, no. 14, pp. 1014-1020, 2009.

[43] R. V. Rao, "A decision making methodology for material selection using an improved compromise ranking method," Materials \& Design, vol. 29, no. 10, pp. 1949-1954, 2008.

[44] R. M. Rodríguez, L. Martínez, V. Torra, Z. S. Xu, and F. Herrera, "Hesitant fuzzy sets: state of the art and future directions," International Journal of Intelligent Systems, vol. 29, no. 6, pp. 495-524, 2014.

[45] R. M. Rodriguez, L. Martínez, and F. Herrera, "Hesitant fuzzy linguistic term sets for decision making," IEEE Transactions on Fuzzy Systems, vol. 20, no. 1, pp. 109-119, 2012.

[46] R. M. Rodríguez, B. Bedregal, H. Bustince et al., "A position and perspective analysis of hesitant fuzzy sets on information fusion in decision making. Towards high quality progress," Information Fusion, vol. 29, pp. 89-97, 2016. 

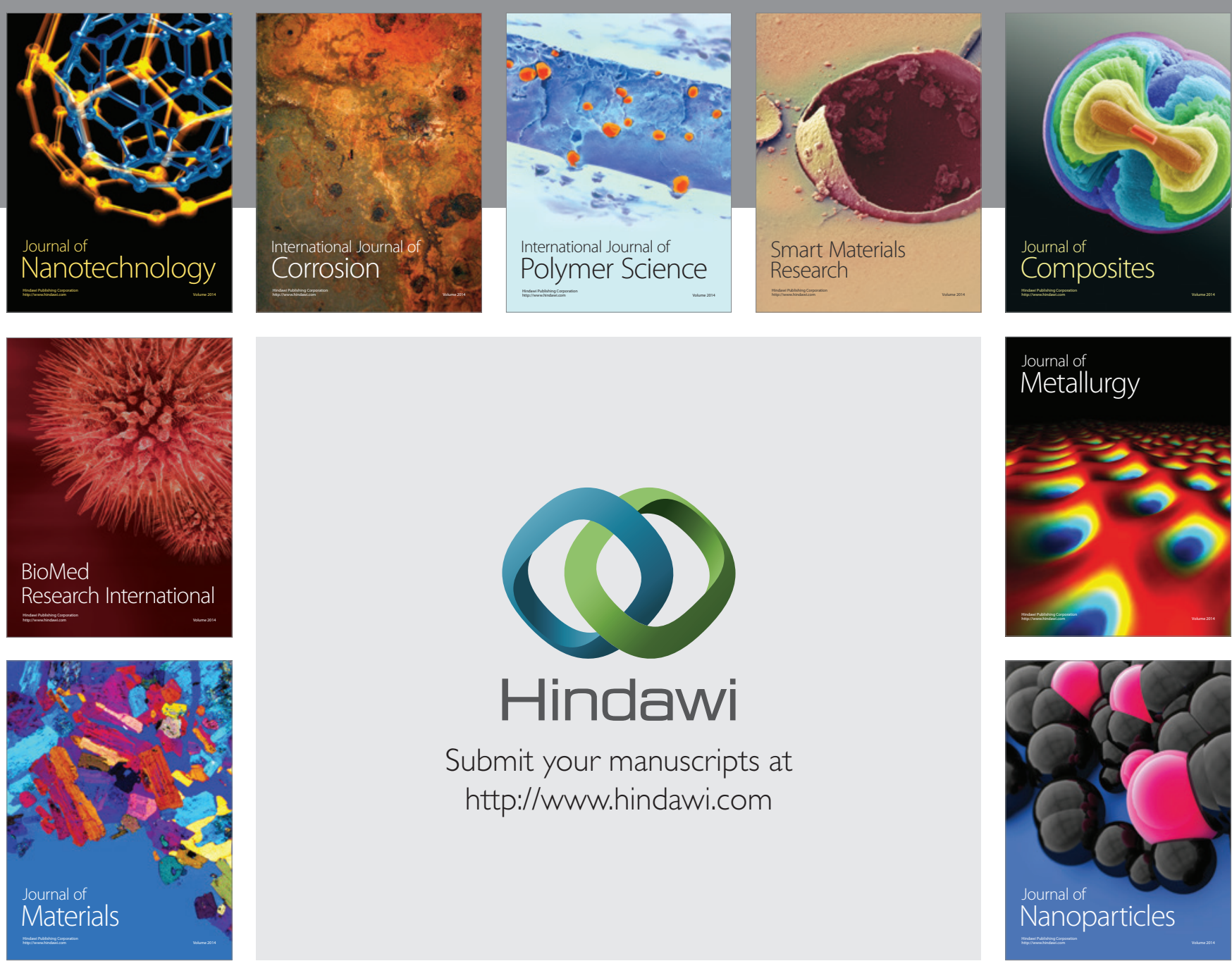

\section{Hindawi}

Submit your manuscripts at

http://www.hindawi.com

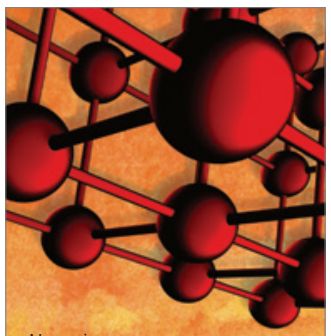

Materials Science and Engineering
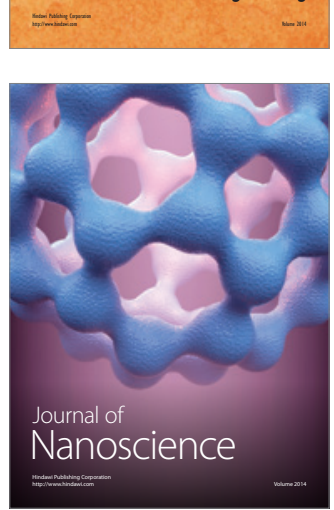
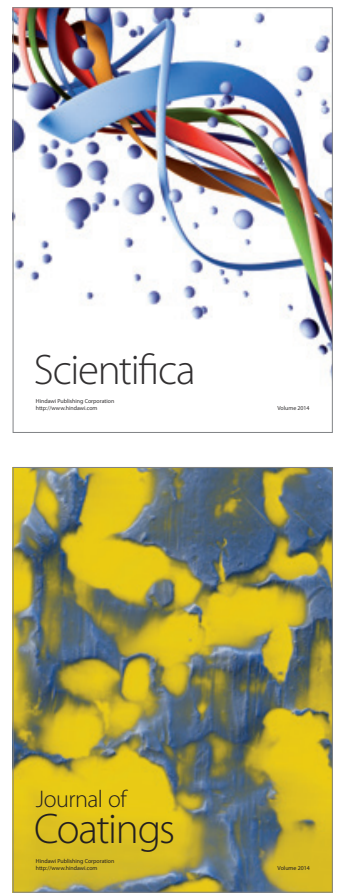
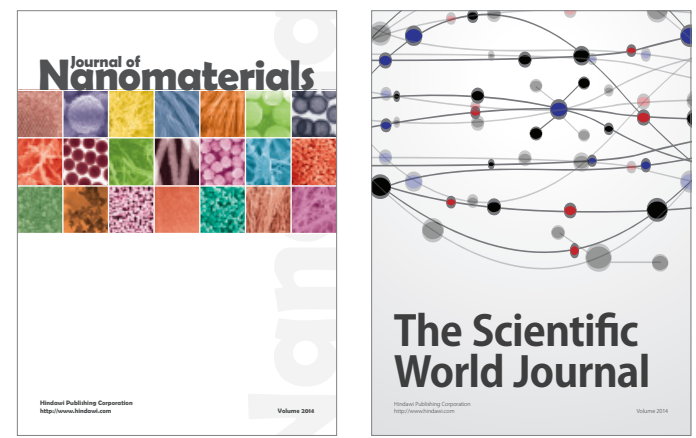

The Scientific World Journal
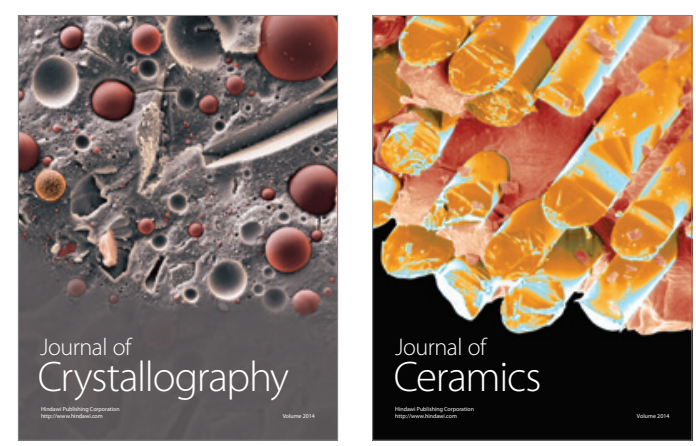
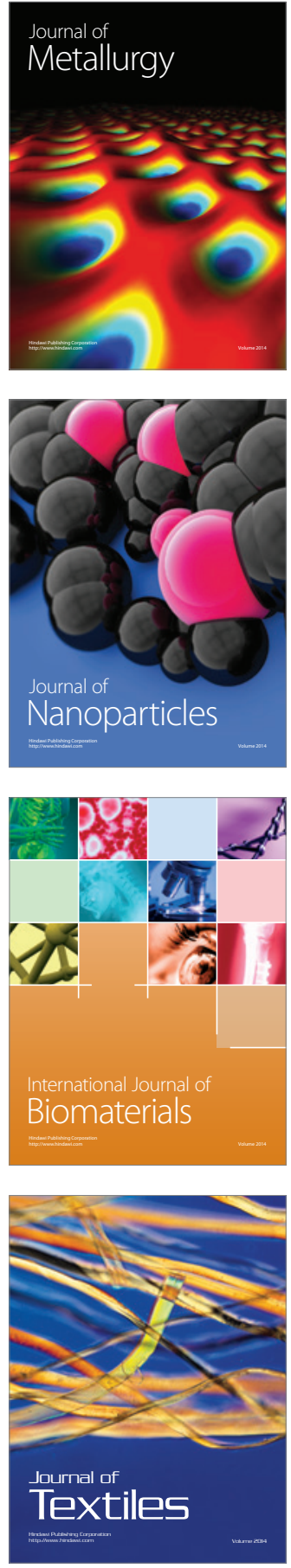